\title{
On low rank classical groups in string theory, gauge theory and matrix models
}

\section{Citation}

Intriligator, Ken, Per Kraus, Anton V Ryzhov, Masaki Shigemori, and Cumrun Vafa. 2004. "On Low Rank Classical Groups in String Theory, Gauge Theory and Matrix Models." Nuclear Physics B 682 (1-2): 45-82. https://doi.org/10.1016/j.nuclphysb.2003.12.030.

\section{Permanent link}

http://nrs.harvard.edu/urn-3:HUL.InstRepos:41385089

\section{Terms of Use}

This article was downloaded from Harvard University's DASH repository, and is made available under the terms and conditions applicable to Other Posted Material, as set forth at http:// nrs.harvard.edu/urn-3:HUL.InstRepos:dash.current.terms-of-use\#LAA

\section{Share Your Story}

The Harvard community has made this article openly available.

Please share how this access benefits you. Submit a story.

Accessibility 
hep-th/0311181

BRX TH-525

HUTP-03/A073

UCLA-03-TEP-29

UCSD-PTH-03-14

\title{
On Low Rank Classical Groups in String Theory, Gauge Theory and Matrix Models
}

\author{
Ken Intriligator ${ }^{1}$, Per Kraus ${ }^{2}$, Anton V. Ryzhov ${ }^{3}$, Masaki Shigemori ${ }^{2}$ and Cumrun Vafa ${ }^{4}$ \\ 1 Department of Physics, University of California, San Diego, La Jolla, CA 92093-0354, USA \\ 2 Department of Physics and Astronomy, UCLA, Los Angeles, CA 90095-1547, USA \\ 3 Department of Physics, Brandeis University, Waltham, MA 02454, USA \\ 4 Jefferson Physical Laboratory, Harvard University, Cambridge, MA 02138, USA
}

\begin{abstract}
We consider $\mathcal{N}=1$ supersymmetric $U(N), S O(N)$, and $S p(N)$ gauge theories, with two-index tensor matter and added tree-level superpotential, for general breaking patterns of the gauge group. By considering the string theory realization and geometric transitions, we clarify when glueball superfields should be included and extremized, or rather set to zero; this issue arises for unbroken group factors of low rank. The string theory results, which are equivalent to those of the matrix model, refer to a particular UV completion of the gauge theory, which could differ from conventional gauge theory results by residual instanton effects. Often, however, these effects exhibit miraculous cancellations, and the string theory or matrix model results end up agreeing with standard gauge theory. In particular, these string theory considerations explain and remove some apparent discrepancies between gauge theories and matrix models in the literature.
\end{abstract}

November, 2003 


\section{Introduction}

Large $N$ topological string duality [1] embedded in superstrings [2, [3] has led to a new perspective on $\mathcal{N}=1$ supersymmetric gauge theories: that the exact effective superpotential can be efficiently computed by including glueball fields. For example, in a theory with gauge group $G$, with tree-level superpotential leading to a breaking pattern

$$
G(N) \rightarrow \prod_{i=1}^{K} G_{i}\left(N_{i}\right)
$$

the dynamics is efficiently encoded in a superpotential $W_{\text {eff }}\left(S_{1}, \ldots S_{K} ; g_{j}, \Lambda\right)\left(g_{j}\right.$ are the parameters in $W_{\text {tree }}$ and $\Lambda$ is the dynamical scale). Further, string theory implies [3]

$$
W_{\mathrm{eff}}\left(S_{i} ; g_{j}, \Lambda\right)=\sum_{i=1}^{K}\left(h_{i} \frac{\partial \mathcal{F}\left(S_{i}\right)}{\partial S_{i}}-2 \pi i \tau_{i} S_{i}\right),
$$

with $h_{i}$ and $\tau_{i}$ the fluxes through $A_{i}$ and $B_{i}$ three-cycles in the geometry, as will be reviewed in sect. 3. The prepotential $\mathcal{F}\left(S_{i}\right)$ in (1.2) is computable in terms of geometric period integrals, which yields [3]

$$
\frac{\partial \mathcal{F}\left(S_{i}\right)}{\partial S_{i}}=S_{i}\left(\log \left(\frac{\Lambda_{i}^{3}}{S_{i}}\right)+1\right)+\frac{\partial}{\partial S_{i}} \sum_{i_{1}, \ldots, i_{K} \geq 0} c_{i_{1} \ldots i_{K}} S_{1}^{i_{1}} \cdots S_{K}^{i_{k}}
$$

with coefficients $c_{i_{1} \ldots i_{K}}$ depending on the $g_{j}$ (but not on the gauge theory scale $\Lambda$ ). In [4] it was shown how planar diagrams of an associated matrix model can also be used to compute (1.2) and (1.3). Based on the stringy examples, this was generalized in [5] to a more general principle to gain non-perturbative information about the strong coupling dynamics of gauge theories, by extremizing the perturbatively computed glueball superpotential.

There are two aspects to the above statements: first that the glueball fields $S_{i}$ are the 'right' variables to describe the IR physics, and second that perturbative gauge theory techniques suffice to compute the glueball superpotential. The latter statement has now been proven in two different approaches for low powers of the glueball fields $S_{i}$ in (1.3) [6, [0. For powers of the glueball fields $S_{i}$ larger than the dual Coxeter number of the group, an ambiguity sets in for the glueball computation of the coefficients $c_{i_{1} \ldots i_{K}}$ in both of these approaches. The matrix model provides a natural prescription for how to resolve this ambiguity, essentially by continuing from large $N_{i}$. It was argued in [8.9] that the string geometry / matrix model result (since the string geometry and matrix model results

are identical, we refer to them synonymously) has the following meaning: it computes the $F$-terms for different supersymmetric gauge theories, which can be expressed in terms of $G(N+k \mid k)$ supergroups. The $W_{\text {eff }}\left(S_{i}\right)$ is independent of $k$, and the above ambiguity can 
be eliminated by taking $k$, and hence the dual Coxeter number, arbitrarily large. The $G(N)$ theory of interest is obtained from the $G(N+k \mid k)$ theory by Higgsing; but there can be residual instanton contributions to $W_{\text {eff }}$ [9], which can lead to apparent discrepancies between the matrix model and gauge theory results. We will somewhat clarify here when such residual instanton effects do, or do not, lead to discrepancies with standard gauge theory results.

There is another, more non-trivial assumption in [5]: the statement that the glueball fields $S_{i}$ are the 'right' variables in the IR. This assumption was motivated from the string dualities $[1 / 30$, where geometric transition provide the explanation of why the glueball fields are the natural IR variables: heuristically, $\left\langle S_{i}\right\rangle$ corresponds to confinement. However, this is not quite correct: it also applies to abelian theories, as had been noted in [3]. So the deep explanation of why we should choose certain dynamical $S$ variables remains mysterious.

In this paper, we will uncover the precise prescription for the correct choice of IR variables. This will be done from the string theory perspective, by arguing in which cases there is a geometric transition in string theory. For the general breaking pattern (1.1), our prescription for treating the glueball field $S_{i}$, corresponding to the factor $G_{i}$ in (1.1), is as follows: If $h\left(G_{i}\right)>0$ we include $S_{i}$ and extremize $W_{\mathrm{eff}}\left(S_{i}\right)$ with respect to it. On the other hand, if $h\left(G_{i}\right) \leq 0$ we do not include or extremize $S_{i}$, instead we just set $S_{i} \rightarrow 0$. Here we define the generalized dual-Coxeter numbers 1

$$
\begin{aligned}
h(U(N)) & =N, \\
h(S p(N)) & =N+1, \\
h(S O(N)) & =N-2,
\end{aligned}
$$

which are generalized in that (1.4) applies for all $N \geq 0$. In particular $h(U(1))=$ $h(S p(0))=1$, so when some $G_{i}$ factor in (1.1) is $U(1)$ or $S p(0)$, our prescription is to include the corresponding $S_{i}$ and extremize with respect to it. On the other hand, $h(U(0))=0$ and $h(S O(2))=0$, so when some $G_{i}$ factor in (1.1) is $U(0)$ or $S O(2)$, our prescription is to just set the corresponding $S_{i}=0$ from the outset. (Note that $U(1)$ and $S O(2)$ are treated differently here.)

This investigation was motivated by trying to understand the discrepancies found in [10] for $S p(N)$ theory with antisymmetric tensor matter, where the superpotentials from the matrix model and gauge theory were found to differ at order $h$ in perturbation theory and beyond. The analysis considered the trivial breaking pattern $S p(N) \rightarrow S p(N)$ and a single glueball was introduced corresponding to the single unbroken gauge group factor. In [9], various gauge theories including this example were studied, and an explanation for the discrepancies was proposed in terms of the conjecture, mentioned above, that

1 Our convention is such that $S p(N) \subset S U(2 N)$, and hence $S p(1) \cong S U(2)$. 
the string theory / matrix model actually computes the superpotential of the large $k$ $G(N+k \mid k)$ supergroup theories, rather than the ordinary $G(N)$ theory. In this context, the trivial breaking pattern considered in [10] should be understood as $S p(N) \rightarrow S p(N) \times$ $S p(0)$, which is completed to $S p(N+k \mid k) \rightarrow S p\left(N+k_{1} \mid k_{1}\right) \times S p\left(k_{2} \mid k_{2}\right)$. In particular, $S p(0)$ factors, while trivial in standard gauge theory, are non-trivial in the string theory geometry / matrix model context: there can be a residual instanton contribution to the superpotential when one Higgses $S p\left(k_{2} \mid k_{2}\right)$ down to $S p(0 \mid 0)=S p(0)$, as explicitly seen in [9] for the case of breaking $S p(0) \rightarrow S p(0)$ with quadratic $W_{\text {tree }}$ 日. Related aspects of "Sp(0)" being non-trivial in the string / matrix model context were subsequently discussed in $11,12,13$.

However, it turns out that one also needs to modify the matrix model side of the computation to take into account the $S p(0)$ factors. This was found by Cachazo [11, who showed that the loop equations determining $T(z) \equiv \operatorname{Tr}\left(\frac{1}{z-\Phi}\right)$ and $R(z) \equiv-\frac{1}{32 \pi^{2}} \operatorname{Tr}\left(\frac{W_{\alpha} W^{\alpha}}{z-\Phi}\right)$ for the $S p(N)$ theory with antisymmetric tensor matter [14,15] could be related to those of a $U(2 N+2 K)$ gauge theory with adjoint matter, with $S p(N) \rightarrow S p(N) \times S p(0)^{K-1}$ mapped to $U(2 N+2 K) \rightarrow U(2 N+2) \times U(2)^{K-1}$. It was thus shown in [11] that vanishing period of $T(z) d z$ through a given cut, corresponding to an $S p(0)$ factor, does not imply that the cut closes up on shell (aspects of the periods in this theory were also discussed in [16]). This fits with our above prescription that the $S p(0)$ glueballs should be included and extremized in the string theory / matrix model picture, as would be done for $U(2)$, rather than set to zero, as was originally done in [10]. We stress that we are not yet even discussing whether or not the string theory / matrix model result agrees with standard gauge theory. Irrespective of any comparison with standard gauge theory, the prescription to obtain the actual string theory / matrix model result is as described above (1.4). Having obtained that result, we can now discuss comparisons with standard gauge theory results. As seen in [11], by solving the $U(2 N+2 K)$ loop equations for the present case, this corrected matrix model result now agrees perfectly with standard gauge theory! This will be discussed further here, with all glueball fields $S_{i}$ included.

This agreement, between the matrix model result and standard gauge theory, is in a sense surprising for this particular theory, in light of the $S p(k \mid k)$ description of [9] for the unbroken $S p(0)$ factors, with the resulting residual instanton contributions to the

2 It was suggested in the original version of [9] that such $S p(0)$ residual instanton contributions could also play a role for the case of cubic and higher order $W_{\text {tree }}$ (where they had not yet been fully computed) and could explain the apparent matrix model vs. standard gauge theory discrepancies found in [10]. As we will discuss, we now know that this last speculation was not correct. The corrected proposal of [9] is still that the matrix model computes the superpotential of the $G(N+k \mid k)$ theory, but where the matrix model side of the computation should be corrected, as we discuss in this paper, to include glueball fields for the $S p(0)$ factors. 
superpotential. As we will explain later in this paper, the agreement here between matrix models and standard gauge theory is thanks to a remarkable cancellation of the residual instanton effect terms, which could have spoiled the agreement. The cancellation occurs upon summing over the $i$ in (1.2) from $i=1 \ldots K$.

There are similar remarkable cancellations of the "residual instanton contributions" to the superpotential in many other examples, which we will also discuss. In fact, in all cases that we know of, the only cases where the residual instantons do not cancel is when the gauge theory clearly has some ambiguity, requiring a choice of how to define the theory in the UV; string theory / matrix model gives a particular such choice. Examples of such cases is when the LHS of (1.1) is itself $U(1)$ or $S p(0)$ super Yang-Mills, as discussed in [9]. Other examples where the residual instanton contributions do not cancel, is when the superpotential is of high enough order such that not all operators appearing in it are independent, e.g. terms like $\operatorname{Tr} \Phi^{n}$, for a $U(N)$ adjoint $\Phi$, when $n>N$. In standard gauge theory, there are then potential ambiguities involved in reducing such composite operators to the independent operators, since classical operator identities can receive quantum corrections. The residual instanton contributions, which do not cancel generally in these cases, imply specific quantum relations for these operators, corresponding to the specific UV completion. See 17, 18 for related issues.

The organization of this paper is as follows: In section 2 we summarize the gauge theories under consideration. In section 3 we review the type IIB string theory construction of these gauge theories. We also discuss maps of the exact superpotentials of $S p$ and $S O$ theories to those of $U$ theories, generalizing observations of [19,20,21, 11, 13, In section 4 we explain, from the string theory perspective in which cases we have a geometric transition. In section 5 we consider examples, where the glueball fields $S_{i}$ of all group factors are correctly accounted for on the matrix model side. The results thereby obtained via matrix models are found to agree with those of standard gauge theory. In many of these examples, this agreement relies on a remarkable interplay of different residual instanton contributions, which sometimes fully cancel. Residual instantons are discussed further in sect. 6, with examples illustrating cases where they do, or do not, cancel. In appendix A, a proof of a general relation between the $S^{2}$ and $R P^{2}$ contributions to the matrix model free energy is given, and also the matrix model computation of superpotential is presented. In appendix $\mathrm{B}$ the gauge theory computation of the superpotential is discussed.

\section{The gauge theory examples}

The specific examples of $\mathcal{N}=1$ supersymmetric gauge theories which we consider, 
with breaking patterns as in (1.1), are as follows:

$\begin{array}{ccc}U(N) & \text { with adjoint } \Phi & U(N) \rightarrow \prod_{i=1}^{K} U\left(N_{i}\right), \\ S O(N) & \text { with adjoint } \Phi: & S O(N) \rightarrow S O\left(N_{0}\right) \times \prod_{i=1}^{K} U\left(N_{i}\right), \\ S p(N) & \text { with adjoint } \Phi: & S p(N) \rightarrow S p\left(N_{0}\right) \times \prod_{i=1}^{K} U\left(N_{i}\right), \\ S O(N) & \text { with symmetric } S: & S O(N) \rightarrow \prod_{i=1}^{K} S O\left(N_{i}\right), \\ S p(N) & \text { with antisymmetric } A: & S p(N) \rightarrow \prod_{i=1}^{K} S p\left(N_{i}\right), \\ U(N) & \text { with } \Phi+S+\widetilde{S}: & U(N) \rightarrow S O\left(N_{0}\right) \times \prod_{i=1}^{K} U\left(N_{i}\right), \\ U(N) & \text { with } \Phi+A+\widetilde{A}: & U(N) \rightarrow S p\left(N_{0}\right) \times \prod_{i=1}^{K} U\left(N_{i}\right) .\end{array}$

For $U(N)$ with adjoint $\Phi$, the tree-level superpotential is taken to be

$$
W_{\text {tree }}=\operatorname{Tr}[W(\Phi)], \quad W(x)=\sum_{j=1}^{K+1} \frac{g_{j}}{j} x^{j},
$$

with $K$ potential wells. In the classical vacua, with breaking pattern as in (2.1), $\Phi$ has $N_{i}$ eigenvalues equal to the root $a_{i}$ of

$$
W^{\prime}(x)=\sum_{j=1}^{K+1} g_{j} x^{j-1} \equiv g_{K+1} \prod_{i=1}^{K}\left(x-a_{i}\right),
$$

with $\sum_{i=1}^{K} N_{i}=N$. For $S O(N)$ with symmetric tensor or $S p(N)$ with antisymmetric tensor we take

$$
W_{\text {tree }}=\frac{1}{2} \operatorname{Tr} W(S), \quad \text { or } \quad W_{\text {tree }}=\frac{1}{2} \operatorname{Tr} W(A),
$$

respectively, where $W(x)$ is as in (2.2), the factor of $\frac{1}{2}$ is for convenience, because the eigenvalues of $S$ or $A$ appear in pairs, and the indices are contracted with $\delta_{b}^{a}$ for $S O(N)$ or $J_{b}^{a}$ for $S p(N)$. For $S O(N)$ and $S p(N)$ with adjoint matter, the tree-level superpotential is

$$
W_{\text {tree }}=\frac{1}{2} \operatorname{Tr}[W(\Phi)], \quad W(x)=\sum_{j=1}^{K+1} \frac{g_{2 j}}{2 j} x^{2 j}
$$

since all Casimirs of the adjoint $\Phi$ are even, and the $\frac{1}{2}$ is again for convenience because the eigenvalues appear in pairs. $\Phi$ 's eigenvalues sit at the zeros of

$$
W^{\prime}(x)=\sum_{j=1}^{K+1} g_{2 j} x^{2 j-1} \equiv g_{2 K+2} x \prod_{i=1}^{K}\left(x^{2}-a_{i}^{2}\right) .
$$

The breaking pattern in (2.1) has $N_{0}$ eigenvalues of $\Phi$ equal to zero, and $N_{i}$ pairs at $\pm a_{i}$, so $N=N_{0}+\sum_{i=1}^{K} 2 N_{i}$ for $S O(N) \rightarrow S O\left(N_{0}\right) \times \prod_{i=1}^{K} U\left(N_{i}\right)$, and $N=N_{0}+\sum_{i=1}^{K} N_{i}$ for $S p(N) \rightarrow S p\left(N_{0}\right) \times \prod_{i=1}^{K} U\left(N_{i}\right)$ (with the convention $\left.S p(1) \cong S U(2)\right)$. 
The next to last example in (2.1) is the $\mathcal{N}=2 U(N)$ theory with a matter hypermultiplet in the two-index symmetric tensor representation, breaking $\mathcal{N}=2$ to $\mathcal{N}=1$ by a superpotential as in (2.2):

$$
W=\sum_{j=1}^{K+1} \frac{g_{j}}{j} \operatorname{Tr} \Phi^{j}+\sqrt{2} \operatorname{Tr} \widetilde{S} \Phi S .
$$

In addition to the possibility of $\Phi$ 's eigenvalues sitting in any of the $K$ critical points $W^{\prime}(x)$ analogous to (2.3), there is a vacuum where $N_{0}$ eigenvalues sits at $\phi=0$, with $\langle S \widetilde{S}\rangle \neq 0$, breaking $U\left(N_{0}\right) \rightarrow S O\left(N_{0}\right)$. The last example in (2.1) is the similar theory where the $\mathcal{N}=2$ hypermultiplet is instead in the antisymmetric tensor representation $A$, rather than the symmetric tensor $S$. These last two classes of examples were considered in [22, 23, 24].

In all of these theories, the low energy superpotential is of the general form

$$
W_{\text {low }}\left(g_{j}, \Lambda\right)=W_{\mathrm{cl}}\left(g_{j}\right)+W_{\mathrm{gc}}\left(\Lambda_{i}\right)+W_{H}\left(g_{j}, \Lambda\right) .
$$

$W_{\mathrm{cl}}\left(g_{j}\right)$ is the classical contribution (evaluating $W_{\text {tree }}$ in the appropriate minima). $W_{\mathrm{gc}}\left(\Lambda_{i}\right)$ is the gaugino condensation contribution in the unbroken gauge groups of (1.1),

$$
W_{\mathrm{gc}}\left(\Lambda_{j}\right)=\sum_{i=1}^{K} h_{i} e^{2 \pi i n_{i} / h_{i}} \Lambda_{i}^{3},
$$

where $h_{i}=C_{2}\left(G_{i}\right)$ is the dual Coxeter number of the group $G_{i}$ in (1.1), with the phase factors associated with the $\mathbf{Z}_{2 h_{i}} \rightarrow \mathbf{Z}_{2}$ chiral symmetry breaking of the low-energy $G_{i}$ gaugino condensation. The scales $\Lambda_{i}$ are related to $\Lambda$ by threshold matching for the fields which got a mass from $W_{\text {tree }}$ and the breaking (1.1); some examples, with breaking patterns as in (2.1), are as follows. For $U(N)$ with adjoint $\Phi$ :

$$
\Lambda_{i}^{3 N_{i}}=\Lambda^{2 N} W^{\prime \prime}\left(a_{i}\right)^{N_{i}} \prod_{j \neq i} m_{W_{i j}}^{-2 N_{j}}=g_{K+1}^{N_{i}} \Lambda^{2 N} \prod_{j \neq i}\left(a_{j}-a_{i}\right)^{N_{i}-2 N_{j}} .
$$

For $S O(N)$ with adjoint, breaking $S O(N) \rightarrow S O\left(N_{0}\right) \times \prod_{i=1}^{K} U\left(N_{i}\right)$,

$$
\begin{aligned}
\Lambda_{0}^{3\left(N_{0}-2\right)} & =g_{2 K+2}^{N_{0}-2} \Lambda^{2(N-2)} \prod_{i=1}^{K} a_{i}^{2\left(N_{0}-2\right)-4 N_{i}} \\
\Lambda_{i}^{3 N_{i}} & =2^{-N_{i}} g_{2 K+2}^{N_{i}} \Lambda^{2(N-2)} a_{i}^{-2\left(N_{0}-2\right)} \prod_{j \neq i}^{K}\left(a_{i}^{2}-a_{j}^{2}\right)^{N_{i}-2 N_{j}} .
\end{aligned}
$$

For $S p(N)$ with adjoint

$$
\begin{aligned}
\Lambda_{0}^{3\left(N_{0}+1\right)} & =g_{2 K+2}^{N_{0}+1} \Lambda^{2(N+1)} \prod_{i=1}^{K} a_{i}^{2\left(N_{0}+1\right)-2 N_{i}} \\
\Lambda_{i}^{3 N_{i}} & =2^{-N_{i}} g_{2 K+2}^{N_{i}} \Lambda^{4(N+1)} a_{i}^{-4\left(N_{0}+1\right)} \prod_{j \neq i}^{K}\left(a_{i}^{2}-a_{j}^{2}\right)^{N_{i}-2 N_{j}}
\end{aligned}
$$


the $U\left(N_{i}\right)$ has index of embedding 2, which is why the $U\left(N_{i}\right)$ one-instanton factor is related to the $S p(N)$ two-instanton factor in (2.12). For $S O(N)$ with symmetric tensor,

$$
\Lambda_{i}^{3\left(N_{i}-2\right)}=g_{K+1}^{N_{i}+2} \Lambda^{2 N-8} \prod_{j \neq i=1}^{K}\left(a_{i}-a_{j}\right)^{N_{i}+2-2 N_{j}}
$$

For $S p(N)$ with antisymmetric $A$ :

$$
\Lambda_{i}^{3\left(N_{i}+1\right)}=g_{K+1}^{N_{i}-1} \Lambda^{2 N+4} \prod_{j \neq i}^{K}\left(a_{i}-a_{j}\right)^{N_{i}-1-2 N_{j}} .
$$

Finally, the term $W_{H}\left(g_{j}, \Lambda\right)$ in (2.8) are additional non-perturbative contributions, which can be regarded as coming from the massive, broken parts of the gauge group.

In the description with the glueballs $S_{i}$ integrated in, as in (1.2), the gaugino condensation contribution comes from the first term in (1.3):

$$
W_{\mathrm{gc}}\left(S_{i}, \Lambda\right)=\sum_{i=1}^{K} h_{i} S_{i}\left(\log \left(\frac{\Lambda_{i}^{3}}{S_{i}}\right)+1\right)
$$

and $W_{H}\left(g_{i}, \Lambda\right)$ comes from the last terms in (1.3), upon integrating out the $S_{i}$. When the minima $a_{i}$ of the superpotential are widely separated, the contributions $W_{H}$ from these last terms are subleading as compared with $W_{\mathrm{gc}}$. As in (1.2), the full glueball superpotential (1.2) can be computed via the string theory geometric transition, in terms of certain period integrals [2. [3], as will be reviewed in the next section, or via the matrix models. In that context, the term $W_{\mathrm{gc}}\left(S_{i}, \Lambda_{i}\right)$ comes from the integration measure (as is also natural in field theory, since it incorporates the $U(1)_{R}$ anomaly) and $W_{H}\left(S_{i}, g_{j}\right)$ can be computed perturbatively [4.5]. The perturbative computation of $W_{H}\left(S_{i}, g_{j}\right)$ can also be understood directly in the gauge theory [6], up to ambiguities in terms $S^{n}$ with $n>h=C_{2}(G)$. The string theory/ matrix model constructions yield a specific way of resolving these ambiguities, which correspond to a particular UV completion of the gauge theory [8,9].

As discussed in the introduction, our present interest will be in analyzing this circle of ideas when some of the gauge group factors in (1.1) are of low rank, or would naively appear to be trivial, e.g. $U(1), U(0), S O(2), S O(0)$, and $S p(0)$.

\section{Geometric transition of $U(N)$ and $\operatorname{SO} / \operatorname{Sp}(N)$ theories}

In this section we briefly review the type IIB geometric engineering of relevant $U(N)$ and $S O / S p(N)$ theories, and their geometric transition. 
3.1. $U(N)$ with adjoint and $W_{\text {tree }}=\operatorname{Tr} \sum_{j=1}^{K+1} \frac{g_{i}}{j} \Phi^{j}$

The Calabi-Yau geometry relevant to this theory [3] is the non-compact $A_{1}$ fibration

$$
W^{\prime}(x)^{2}+y^{2}+u^{2}+v^{2}=0
$$

This fibration has $K$ conifold singularities at the critical points of $W(x)$, i.e. at $W^{\prime}(x)=$ 0 . Near each of the singularities, the geometry (3.1) is the same as the usual conifold $x^{\prime 2}+y^{2}+u^{2}+v^{2}=0$, which is topologically a cone with base $S^{2} \times S^{3}$.

The singularities can be resolved by blowing up a 2-sphere $S^{2}=\mathbf{P}^{1}$ at each singularity. We can realize the $U(N)$ gauge theory with adjoint matter and superpotential (2.2) in type IIB superstring theory compactified on this resolved geometry, with $N$ D5-branes partially wrapping the $K \mathbf{P}^{1}$ 's. The gauge theory degrees of freedom correspond to the open strings living on these D5-branes. The classical supersymmetric vacuum is obtained by distributing the $N_{i}$ D5-branes over the $i$-th 2 -sphere $\mathbf{P}_{i}^{1}$ with $i=1, \cdots, K$. The corresponding breaking pattern of the gauge group is as in (2.1): $U(N) \rightarrow \prod_{i=1}^{K} U\left(N_{i}\right)$.

At low energy, the gauge theory confines (when $N_{i}>1$ ), each $U\left(N_{i}\right)$ factor developing nonzero vev of the glueball superfield $S_{i}$. In string theory this is described by the geometric transition [1,2, 3] in which the resolved conifold geometry with $\mathbf{P}^{1}$ 's wrapped by D5-branes is replaced by a deformed conifold geometry

$$
W^{\prime}(x)^{2}+f_{K-1}(x)+y^{2}+u^{2}+v^{2}=0,
$$

where $f_{K-1}(x)$ is a polynomial of degree $K-1$ in $x$ and parametrizes the deformation. After the geometric transition, each 2 -sphere $\mathbf{P}_{i}^{1}$ wrapped by $N_{i}$ D5-branes is replaced by a 3-sphere $A_{i}$ with 3 -form RR flux through it:

$$
\oint_{A_{i}} H=N_{i}
$$

where $H=H_{R R}+\tau H_{N S}$ and $\tau=C^{(0)}+i e^{-\Phi}$ is the complexified coupling constant of type IIB theory. We define the periods of the Calabi-Yau geometry (3.2) by

$$
S_{i} \equiv \frac{1}{2 \pi i} \oint_{A_{i}} \Omega, \quad \Pi_{i} \equiv \int_{B_{i}}^{\Lambda_{b}} \Omega,
$$

where $\Omega$ is the holomorphic 3 -form, $B_{i}$ is the noncompact 3 -cycle dual to the 3 -cycle $A_{i}$, and $\Lambda_{b}$ is a cutoff needed to regulate the divergent $B_{i}$ integrals. The IR cutoff $\Lambda_{b}$ is to be identified with the UV cutoff of the $4 \mathrm{~d}$ gauge theory. The set of variables $S_{i}$ measure the size of the blown up 3-spheres, and can be used to parametrize the deformation in place of the $K$ coefficients of the polynomial $f_{K-1}(x)$. 
The dual theory after the geometric transition is described by a $4 \mathrm{~d}, \mathcal{N}=1 U(1)^{K}$ gauge theory, with $K U(1)$ vector superfields $V_{i}$ and $K$ chiral superfields $S_{i}$. If not for the fluxes, this theory would be $\mathcal{N}=2$ supersymmetric $U(1)^{K}$, with $\left(V_{i}, S_{i}\right)$ the $\mathcal{N}=2$ vector super-multiplets, $S_{i}$ being the Coulomb branch moduli. This low-energy $U(1)^{K}$ theory has a non-trivial prepotential $\mathcal{F}\left(S_{i}\right)$ and the dual periods $\Pi_{i}$ in (3.4) can be written as

$$
\Pi_{i}(S)=\frac{\partial \mathcal{F}}{\partial S_{i}}
$$

Without the fluxes, this prepotential can be understood as coming from integrating out D3 branes which wrap the $A_{i}$ cycles, and are charged under the low-energy $U(1)$ 's [25].

The effect of the added fluxes is to break $\mathcal{N}=2$ supersymmetry to $\mathcal{N}=1$ by the added superpotential 26,27]

$$
W_{\text {flux }}=\int H \wedge \Omega=\sum_{i}\left(\oint_{A_{i}} H \int_{B_{i}}^{\Lambda_{b}} \Omega-\int_{B_{i}}^{\Lambda_{b}} H \oint_{A_{i}} \Omega\right) .
$$

In the present $U(N)$ case, (3.3) and (3.4) gives

$$
W_{\text {flux }}=\sum_{i=1}^{K}\left(N_{i} \Pi_{i}-2 \pi i \alpha S_{i}\right)
$$

where

$$
\oint_{B_{i}}^{\Lambda_{b}} H=\alpha
$$

is the 3 -form NS flux through the 3 -cycle $B_{i}$ and identified with the bare coupling constant of the gauge theory by

$$
2 \pi i \alpha=\frac{8 \pi^{2}}{g_{b}^{2}}=V
$$

where $V$ is the complexified volume of the $\mathbf{P}^{1}$ 's. The $\mathcal{N}=1 U(1)^{K}$ vector multiplets $V_{i}$ remain massless, but the $S_{i}$ now have a superpotential, which fixes them to sit at discrete vacuum expectation values, where they are massive. The fields $S_{i}$ are identified with the glueballs on the gauge theory side.

The superpotential $W_{\text {flux }}\left(S_{i}\right)$ is the full, exact, effective superpotential in (1.2). As can be verified by explicit calculations [2, 3], the leading contribution to (3.7) is always of the form

$$
W_{\text {flux }} \sim \sum_{i=1}^{K}\left[N_{i} S_{i}\left(1-\ln \left(S_{i} / \Lambda_{i}^{3}\right)\right)-2 \pi i \alpha S_{i}\right]
$$

where $\Lambda_{i}$ is related to the scale $\Lambda_{b}$ via precisely the relation (2.10). This leading term (3.10) is the gaugino condensation part of the superpotential, as in (1.3). 


\section{2. $S O$ and $S p$ theories}

The string theory construction of $S O / S p(N)$ theories can be obtained from the above $U(N)$ construction, by orientifolding the geometry before and after the geometric transition by a certain $\mathbf{Z}_{2}$ action. The geometric construction of $S O / S p(N)$ theory with adjoint was discussed in 28,29,20,30, and in that case the invariance of the geometry (3.2) under the $\mathbf{Z}_{2}$ action requires that the polynomial $W(x)$ be even. The geometric construction of $S O / \operatorname{Sp}(N)$ theory with symmetric/antisymmetric tensor was studied in [31, 32, 13,.

In the classical vacuum of the "parent" $U(2 N)$ theory, the gauge group is broken into a product of $U\left(N_{i}\right)$ groups. When a $U\left(N_{i}\right)$ factor is identified with another $U\left(N_{i}\right)$ by the $\mathbf{Z}_{2}$ orientifold action, they lead to a single $U\left(N_{i}\right)$ factor. When a $U\left(N_{i}\right)$ factor is mapped to itself by the $\mathbf{Z}_{2}$ orientifold action, it becomes an $S O\left(N_{i}\right)$ or $S p\left(\frac{1}{2} N_{i}\right)$, depending on the charge of the orientifold hyperplane. As a result, the classical vacuum of the "daughter" $S O / S p(N)$ theory has gauge group broken as in (2.1), depending on whether the theory is $S O$ or $S p$ with adjoint, or $S O$ with symmetric tensor, or $S p$ with antisymmetric tensor:

$$
S O / S p(N) \rightarrow \prod_{i} G_{i}\left(N_{i}\right), \quad G_{i}=U, S O, \text { or } S p
$$

The 3-form RR fluxes from orientifold hyperplanes makes an additional contribution to the superpotential (3.6), and the flux superpotential can be written as

$$
W_{\text {flux }}=\sum_{i}\left[\hat{N}_{i} \Pi_{i}\left(S_{i}\right)-2 \pi i \eta_{i} \alpha S_{i}\right]
$$

where

$$
\hat{N}_{i}=\left\{\begin{array}{ll}
N_{i} & G_{i}=U\left(N_{i}\right), \\
\frac{1}{2} N_{i} \mp 1 & G_{i}=S O\left(N_{i}\right) / S p\left(\frac{1}{2} N_{i}\right),
\end{array} \quad \eta_{i}= \begin{cases}1 & G_{i}=U, \\
1 / 2 & G_{i}=S O / S p .\end{cases}\right.
$$

$\hat{N}_{i}$ is the net 3-form RR flux through the $A_{i}$ cycle. For $U\left(N_{i}\right)$ and $S p\left(N_{i}\right), \hat{N}_{i}$ in $(3.13)$ is the dual Coxeter number (1.4), while for $S O\left(N_{i}\right)$ it is half 3 the dual Coxeter number (1.4). The $1 / 2$ in (3.13) is because the integration over the $A_{i}$ cycles should be halved due to the $\mathbf{Z}_{2}$ identification.

\subsection{Relations between $S O / S p$ theories and $U(N)$ theories}

The result (3.12), with (3.13), gives the exact superpotential of the $S O / S p$ theories in terms of the same periods $S_{i}$ and $\Pi\left(S_{i}\right)$ as an auxiliary $U$ theory. This was first noted

3 So we get $h$ replaced with $h / 2$ for $S O$ groups in (2.9). While one could absorb the overall factor of 2 into the definition of $\Lambda$, the number of vacua should be $h$ whereas here we apparently get $h / 2$ for $S O$ groups. This is because the we don't see spinors or the $\mathbf{Z}_{2}$ part of the center which acts on them; it's analogous to $U(2 N)$ being restricted to vacua with confinement index 2 . 
in [11] at the level of the Konishi anomaly equation as a map between the resolvents of $S p$ theory with antisymmetric matter and $U$ theory with adjoint matter. In [13], it was generalized to the map between the resolvents of $S O / S p$ theories with two-index tensor matter and $U$ theory with adjoint matter, and string theory interpretation was discussed. In this subsection we will derive this map from the string theory perspective using the flux superpotential (3.12). Furthermore, we will clarify the relation of the superpotential and the scale of the $S O / S p$ theories to those of the $U$ theory. The map between resolvents can be derived from these results. For $S p(N)$ theory with an antisymmetric tensor, the scale relation was obtained in a different way in [11].

As a first example, consider $S O(N)$ with an adjoint, with the breaking pattern as in (2.1). The geometric transition result (3.12) and (3.13) implies that the exact superpotential is the same as for the $U(N-2)$ theory with adjoint, with breaking pattern map

$$
S O(N) \rightarrow S O\left(N_{0}\right) \times \prod_{i=1}^{K} U\left(N_{i}\right) \quad \Longleftrightarrow \quad U(N-2) \rightarrow U\left(N_{0}-2\right) \times \prod_{i=1}^{K} U\left(N_{i}\right)^{2} .
$$

The map between the superpotential is

$$
W_{\text {exact }}^{S O(N)}=\frac{1}{2} W_{\text {exact }}^{U(N-2)}
$$

The $S O(N)$ scale matching relation (2.11) is compatible with the map (3.14), since (2.10) for the theory (3.14) reproduces (2.11).

Likewise, $S p(N)$ with adjoint has the same exact superpotential as for the $U(2 N+2)$ theory with adjoint, with

$$
\begin{gathered}
S p(N) \rightarrow S p\left(N_{0}\right) \times \prod_{i=1}^{K} U\left(N_{i}\right) \quad \Longleftrightarrow \quad U(2 N+2) \rightarrow U\left(2 N_{0}+2\right) \times \prod_{i=1}^{K} U\left(N_{i}\right)^{2}, \\
W_{\text {exact }}^{S p(N)}=\frac{1}{2} W_{\text {exact }}^{U(2 N+2)} .
\end{gathered}
$$

The $S p(N)$ scale matching relations (2.12) follow from the $U(N)$ matching relations (2.10) with the replacement (3.16), with the understanding that the $U(2 N+2)$ and $U\left(2 N_{0}+2\right)$ one-instanton factors correspond to the $S p(N)$ and $S p\left(N_{0}\right)$ two-instanton factors; this is related to the index of the imbedding mentioned after (2.12), and is accounted for by dividing the $U(2 N+2)$ superpotential by two, as above.

Next consider $S p(N)$ with antisymmetric tensor $A$ and breaking pattern as in (2.1). The geometric transition result (3.12) and (3.13) implies that the exact superpotential is the same as for the $U(2 N+2 K)$ theory with adjoint and breaking pattern

$$
S p(N) \rightarrow \prod_{i=1}^{K} S p\left(N_{i}\right) \quad \Longleftrightarrow \quad U(2 N+2 K) \rightarrow \prod_{i=1}^{K} U\left(2 N_{i}+2\right)
$$


In the present case, comparing the matching relations (2.14) for $S p(N)$ with symmetric tensor with the matching relations (2.10) for $U(2 N+2 K)$ with adjoint, for the mapping as in (3.17) requires that the scales of the original $S p(N)$ on the LHS of (3.17) and the $U(2 N+2 K)$ on the RHS of (3.17) be related as

$$
\Lambda_{U(2 N+2 K)}^{2(N+K)}=g_{K+1}^{-2} \Lambda_{S p(N)}^{2 N+4} .
$$

Then the $\Lambda_{i}$ of the unbroken groups on both sides of (3.17) coincide, with the understanding that the $U\left(2 N_{i}+2\right)$ one-instanton factors correspond to the $S p\left(N_{i}\right)$ two-instanton factors, as above. The map between the superpotential is

$$
\begin{aligned}
& W_{\text {exact }}^{S p(N)}=\frac{1}{2}\left[W_{\text {exact }}^{U(2 N+2 K)}-\Delta W_{\mathrm{cl}}\right], \quad \Delta W_{\mathrm{cl}}=2 \sum_{i=1}^{K} W\left(a_{i}\right), \\
& \text { i.e. writing } \quad W_{\text {exact }}=W_{\mathrm{cl}}+W_{\text {quant }}, \quad W_{\text {quant }}^{S p(N)}=\frac{1}{2} W_{\text {quant }}^{U(2 N+2 K)} .
\end{aligned}
$$

Note that, in order for the superpotentials on the two sides of (3.17) to fully coincide, one must compensate the classical mismatch $\Delta W_{\mathrm{cl}}$, since each well is occupied by two additional eigenvalues in the theory on the RHS of (3.17). In the string theory geometric transition realization, this constant shift, which is independent of $N, \Lambda$, and the glueball fields $S_{i}$, is most naturally interpreted as an additive shift of the superpotential on the $S p$ side, which can be regarded as coming from the orientifold planes both before and after the transitions. The classical shift of $\Delta W_{\mathrm{cl}}$ leads to slightly different operator expectation values (as computed via $W_{\text {eff }}\left(g_{p}, \Lambda\right)$ as the generating function) between the $S p$ and $U$ theory, as was seen in the example of [11]. Also, writing the map as in (3.17), we want the vacuum with confinement index 2 [1]. We could equivalently replace the RHS of (3.17) with $U(N+K) \rightarrow \prod_{i=1}^{K} U\left(N_{i}+1\right)$, in which case we would not have to divide by 2 in (3.19).

Likewise, $S O(N)$ with symmetric tensor $S$ has exact superpotential related to that of a $U(N-2 K)$ theory with adjoint as

$$
\begin{gathered}
S O(N) \rightarrow \prod_{i=1}^{K} S O\left(N_{i}\right) \Longleftrightarrow U(N-2 K) \rightarrow \prod_{i=1}^{K} U\left(N_{i}-2\right), \\
W_{\text {exact }}^{S O(N)}=\frac{1}{2}\left[W_{\text {exact }}^{U(N-2 K)}+\Delta W_{\mathrm{cl}}\right], \quad \Delta W_{\mathrm{cl}}=2 \sum_{i=1}^{K} W\left(a_{i}\right) .
\end{gathered}
$$

Comparing the matching relations (2.13) for $S O(N)$ with symmetric tensor with those of (2.10) for $U(N-2 K)$ with adjoint requires that the scales of the original $S O(N)$ on the LHS of (3.20) and those of the $U(N-2 K)$ theory on the RHS be related as

$$
\Lambda_{U(N-2 K)}^{2(N-2 K)}=g_{K+1}^{4} \Lambda_{S O(N)}^{2 N-8} .
$$


Then the $\Lambda_{i}$ of the unbroken groups on both sides of (3.20) coincide. Again, in order for the superpotentials on the two sides of (3.20) to fully coincide, one must correct for the classical mismatch $\Delta W_{\mathrm{cl}}$ coming from the fact that the $U(N-2 K)$ theory has two fewer eigenvalues in each well.

In appendix $\mathrm{A}$ we will discuss these relations from the matrix model viewpoint. In this context, the relation relevant for (3.14) and (3.16) was conjectured in [19,20 based on explicit diagrammatic calculations, and it was proven for the case of unbroken gauge group $N_{0}=N$ in [21]. This will be generalized in Appendix A to all breaking patterns. Likewise, the matrix model relation relevant for (3.17) and (3.20) will be proven in the appendix; this is a generalization of the connection found in [11] for the theories in (3.17) and (3.20).

\section{String theory prescription for low rank}

The discussion of the previous section applies for all $N_{i} \geq 0$. We now discuss under which circumstances one expects a transition in string theory, where $S_{i}^{3}$ 's grows, and therefore an effective glueball field $S_{i}$ should be included in the superpotential. Whether or not there is a geometric transition in string theory is a local question, so each $S_{i}^{3}$ can be studied independently. Near any $S_{i}^{3}$ the local physics is just a conifold singularity, so we only need to consider the case of a conifold singularity.

\subsection{Physics near a conifold singularity}

As we saw, $U(N), S O / S p(N)$ gauge theory can be realized in type IIB theory as the open string theory living on the D5-branes partially wrapped on the exceptional $\mathbf{P}^{1}$ of a resolved conifold geometry. There is a $\mathbf{P}^{1}$ associated to each critical point of the polynomial $W(x)$. By the geometric transition duality [2, 3], this gauge theory is dual to the closed string theory in the deformed conifold geometry where the $\mathbf{P}^{1}$ 's have been blown down and $S^{3}$ 's are blown up instead.

Let us focus on one $\mathbf{P}^{1}$ with $N \geq 0$ D5-branes wrapping it. This corresponds to focusing on one critical point on the gauge theory side. We allow $N=0$, which corresponds to an unoccupied critical point. In the neighborhood, the geometry after the geometric transition is approximately a deformed conifold $x^{2}+y^{2}+z^{2}+w^{2}=\mu$ with a blown up $S^{3}$. The low energy degrees of freedom in the four-dimensional theory are the $\mathcal{N}=1 U(1)$ photon vector superfield $V$ and the $\mathcal{N}=1$ chiral superfield $S$. The bosonic component of $S$ is proportional to $\mu$ and measures the size of the $S^{3}$.

First, consider the case without fluxes. Then the closed string theory has $\mathcal{N}=2$ and there is one $\mathcal{N}=2 U(1)$ vector multiplet $(V, S)$. It is known that as the size of the $S^{3}$ goes to zero there appears an extra massless degree of freedom [25], which corresponds to the 
D3-brane wrapping the $S^{3}$. The mass of the wrapped BPS D3-brane is proportional to the area, $S$, of the $S^{3}$, so the mass becomes zero as the $S^{3}$ shrinks to zero, i.e. as $S \rightarrow 0$. This extra degree of freedom is described as an $\mathcal{N}=2$ hypermultiplet charged under the $U(1)$ (of $V$ ). Let us write this hypermultiplet in $\mathcal{N}=1$ language as $(Q, \tilde{Q})$, where $Q$ and $\tilde{Q}$ are both $\mathcal{N}=1$ chiral superfields with opposite $U(1)$ charges. The $\mathcal{N}=2$ supersymmetry requires the superpotential

$$
W_{Q}=\sqrt{2} Q \tilde{Q} S
$$

which indeed incorporates the above situation that the $Q, \tilde{Q}$ become massless as $S \rightarrow 0$. The $D$-flatness is

$$
|Q|^{2}-|\tilde{Q}|^{2}=0
$$

and the $F$-flatness is

$$
Q S=\tilde{Q} S=Q \tilde{Q}=0
$$

The only solution to these is

$$
Q=\tilde{Q}=0, \quad S: \text { any }
$$

which just means that $S \sim \mu$ is a modulus.

Now let us come back to the case with the fluxes. As reviewed in the last section, the fluxes give rise to a superpotential (3.6) which breaks $\mathcal{N}=2$ to $\mathcal{N}=1$ [26,27]. As in (3.12), the local flux superpotential contribution is

$$
W_{\text {flux }}(S) \simeq \hat{N} S\left[1-\ln \left(S / \Lambda^{3}\right)\right]-2 \pi i \eta \alpha S,
$$

where we just keep the leading order term in (3.12), as in (3.10), with

$$
\hat{N}=\left\{\begin{array}{ll}
N & U(N), \\
N / 2 \mp 1 & S O(N) / S p\left(\frac{1}{2} N\right),
\end{array} \quad \eta= \begin{cases}1 & U(N), \\
1 / 2 & S O(N) / S p\left(\frac{1}{2} N\right) .\end{cases}\right.
$$

The scale $\Lambda$ is written in terms of the bare coupling $\Lambda_{b}$ and the coupling constants in the problem, as before, and $2 \pi i \alpha$ is related to the bare gauge coupling by (3.9).

In the following, we discuss the cases with $\hat{N}=0, \hat{N}>0$ and $\hat{N}<0$ in order.

\section{- $\hat{\mathrm{N}}=\mathbf{0}$ case}

In this case, the total superpotential is simply the sum of (4.1) and (4.5):

$$
W=\sqrt{2} Q \tilde{Q} S-2 \pi i \eta \alpha S
$$

The only solution to the equation of motion is

$$
|Q|^{2}=|\tilde{Q}|^{2}, \quad Q \tilde{Q}=\frac{2 \pi i \eta \alpha}{\sqrt{2}}, \quad S=0
$$


This is consistent with the fact that $\alpha$ is proportional to the volume of the $\mathbf{P}^{1}$, and the D3-brane condensation $\langle Q \tilde{Q}\rangle$ corresponds to the size of the $\mathbf{P}^{1}$. Furthermore, since $\langle S\rangle=0$, the superpotential vanishes: $W=0$. Therefore, for $\hat{N}=0$, i.e. for $U(0)$ and $S O(2)$, geometric transition does not take place and we should set the corresponding glueball field $S \rightarrow 0$ from the beginning.

\section{- $\hat{\mathbf{N}}>\mathbf{0}$ case}

In this case, there is a net RR flux through the $A$-cycle: $\oint_{A} H=\hat{N}$. This means that the D3-brane hypermultiplet $(Q, \tilde{Q})$ is infinitely massive, because the RR flux will induce $\hat{N}$ units of fundamental charge on the D3-brane. Since the D3-brane is wrapping a compact space $S^{3}$, the fundamental charge on it should be canceled by $\hat{N}$ fundamental strings attached to it. Those fundamental strings extend to infinity and thus cost infinite energyl. Therefore, we can forget about $Q, \tilde{Q}$ in this case, and the full superpotential is given just by the flux contribution (4.5). The equation of motion gives

$$
S^{\hat{N}} \simeq \Lambda^{3 \hat{N}} e^{-2 \pi i \eta \alpha}
$$

which corresponds to the confining vacua of the gauge theory. Note that this case includes $U(1)$ and $S p(0)$; these theories have a dual confining description. This may sound a little paradoxical, but is related to the fact that the string theory computes not for the standard $G(N)$ gauge theory but the associated $G(N+k \mid k)$ higher rank gauge theory, which is confining and differs from standard $U(1)$ and $S p(0)$ due to residual instanton effects [9].

\section{- $\hat{\mathbf{N}}<\mathbf{0}$ case}

In this case, the same argument as the $\hat{N}>0$ case tells us that we should not include the D3-brane fields $Q, \tilde{Q}$. Hence the superpotential is just the flux part (4.5), which again leads to

$$
S^{\hat{N}} \simeq \Lambda^{3 \hat{N}} e^{-2 \pi i \eta \alpha} .
$$

However, now (4.10) is physically unacceptable, since $S$ diverges in the weak coupling limit where the bare volume of $\mathbf{P}^{1}$ becomes $V=2 \pi i \alpha \rightarrow \infty\left(g_{b} \rightarrow 0\right)$ - i.e. taking $\mathbf{P}^{1}$ large would lead to $S^{3}$ also being large, which does not make sense geometrically. The resolution is that $S$ cannot be a good variable: the $S^{3}$ does not actually blow up, and $S$ should be set to zero, $S \rightarrow 0$, also for this case. Though $S$ is set to zero, the non-zero flux can lead to a non-zero superpotential contribution $W_{\text {flux }}=\hat{N} \Pi(S \rightarrow 0)$.

Note that the above result concerning the sign of $\hat{N}$ does not mean the gauge theory prefers D5-branes to anti-D5-branes; it just means that one should choose the sign of the

4 This phenomenon is the same as that observed in the context of AdS/CFT [33, 34, 35]. 
NS flux (i.e. the sign of $2 \pi i \alpha$ ) appropriately. If one wraps the $\mathbf{P}^{1}$ with anti-D5-branes, one should flip the sign of the NS flux in order to have a blown up $S^{3}$ (which can be viewed as a generalization of Seiberg duality to $N_{f}=0$ ).

\subsection{General prescription}

Although we focused on the physics around just one $\mathbf{P}^{1}$ in the above, the result is applicable to general cases where we have multiple $\mathbf{P}^{1}$ wrapped with D5-branes, because the geometry near each $\mathbf{P}^{1}$ is identical to the conifold geometry considered above. Therefore, if we replace $\hat{N}$ with $\hat{N}_{i}$, all of the above conclusions carry over.

Once we have understood the physics, we can forget about the D3-brane hypermultiplet $(Q, \tilde{Q})$ and state the result as a general prescription for how string theory treats $U(0)$, $S O(0), S O(2)$, and $S p(0), U(1)$ groups in the geometric dual description:

- $\mathrm{U}(0), \mathrm{SO}(0), \mathrm{SO}(2)$ :

There are no glueball variables associated to these gauge groups, so we should take the corresponding $S \rightarrow 0$.

- All other groups, including $\mathbf{S p}(0)$, and $\mathrm{U}(1)$ :

We should consider and extremize the corresponding glueball field $S$.

This prescription should also be applied when using the matrix model [4, 36,5] to compute the glueball superpotentials.

\section{Examples}

Let us scan over all of the examples of (2.1), considering the vacuum where the gauge group is unbroken, and ask when glueball fields $S_{i}$ for the apparently trivial groups in (2.1) should be set to zero, or included and extremized. For the first three cases in (2.1), $U(N), S O(N)$, and $S p(N)$ with adjoint, the breaking (2.1) is $G \rightarrow G \times U(0)^{K-1}$, and the glueball fields $S_{i}$ for the $U(0)$ factors are to be set to zero. This justifies the analysis of these theories in the unbroken vacua in [37,21]. The next case is $S O(N)$ with a symmetric tensor $S$, where the vacuum with unbroken gauge group is to be understood as $S O(N) \rightarrow$ $S O(N) \times S O(0)^{K-1}$, and again the glueball fields $S_{i}$ for the $S O(0)$ factors are set to zero. This eliminates the Veneziano-Yankielowicz part of the superpotential for $S O(0)$, but the -1 unit of flux associated with each $S O(0)$ does contribute to flux terms $\hat{N}_{i} \Pi_{i}=-\Pi_{i}$ in (3.12), even though this does not contain $S O(0)$ glueballs any more.

The next case is $S p(N)$ with an antisymmetric tensor, where the vacuum with unbro-

ken gauge group is to be understood as $S p(N) \rightarrow S p(N) \times S p(0)^{K-1}$. Unlike the above cases, here we must keep and extremize the $S_{i}$ for the $S p(0)$ factors, as will be further discussed shortly. 
For the next to last example in 2.1$), U(N)$ with $\Phi+S+\widetilde{S}$, the vacuum with unbroken gauge group is to be understood as $U(N) \rightarrow S O(0) \times U(N) \times U(0)^{K-1}$, and the glueball fields $S_{i}$ for $S O(0)$ and $U(0)$ are to be set to zero. Finally, for the last example in (2.1), $U(N)$ with $\Phi+A+\widetilde{A}$, the vacuum with unbroken gauge group is to be understood as $U(N) \rightarrow S p(0) \times U(N) \times U(0)^{K-1}$. Though the string engineering of these examples differs somewhat from those discussed in sect. 4 (it was obtained in [30]), the general prescription of sect. 4 is expected to carry over in general: the glueball field $S_{0}$ for the $S p(0)$ factor should be included and extremized, rather than set to zero. On the other hand, the $S_{i}$ for the $U(0)$ factors are set to zero. These latter two theories in (2.1) were considered in [24] and it was noted there that for the case with antisymmetric one expands on the matrix model side around a different vacuum than would be naively expected; this indeed corresponds to keeping and extremizing the glueball field $S_{0}$ for the $S p(0)$ factor, as we have discussed.

We now illustrate some other breaking patterns in the examples of (2.1), from the matrix model perspective, for the case of $K=2$. We also compare with standard gauge theory results and generally find agreement, even in cases where there was room for disagreement because of the possibility of residual instanton effects along the lines of [9]. As will be discussed in more detail in the following section, the agreement is thanks to a remarkable interplay of different residual instanton contributions.

\section{1. $S O / S p(N)$ theory with adjoint}

Consider $\mathcal{N}=2 S O(2 N) / S p(N)$ theory broken to $\mathcal{N}=1$ by a tree level superpotential for the adjoint chiral superfield $\Phi$ :

$$
W_{\text {tree }}=\frac{1}{2} \operatorname{Tr}[W(\Phi)], \quad W(x)=\frac{m}{2} x^{2}+\frac{g}{4} x^{4} .
$$

In the $S O$ case, we can skew-diagonalize $\Phi$ as

$$
\Phi \sim \operatorname{diag}\left[\lambda_{1}, \cdots, \lambda_{N}\right] \otimes i \sigma^{2} .
$$

The superpotential (5.1) has critical points at $\lambda=0$ and $\lambda= \pm \sqrt{m / g}$. The classical supersymmetric vacuum of the theory is given by distributing $2 N_{0}$ of the $2 N$ "eigenvalues" $\lambda_{i}$ at the critical point $\lambda=0$ and $N_{1}$ "eigenvalue" pairs at $\lambda= \pm \sqrt{m / g}$, with $N_{0}+N_{1}=N$. In this vacuum, the gauge group breaks as $S O(2 N) \rightarrow S O\left(2 N_{0}\right) \times U\left(N_{1}\right)$ or $S p(N) \rightarrow$ $S p\left(N_{0}\right) \times U\left(N_{1}\right)$.

In the matrix model prescription, the effective superpotential in these vacua is calculated by matrix model as

$$
\begin{aligned}
W_{\mathrm{DV}}\left(S_{0}, N_{0} ; S_{1}, N_{1}\right) & =\left(N_{0} \mp 1\right) S_{0}\left[1-\ln \left(S_{0} / \Lambda_{0}^{3}\right)\right]+N_{1} S_{1}\left[1-\ln \left(S_{1} / \Lambda_{1}^{3}\right)\right]+W_{\text {pert }} \\
W_{\text {pert }} & =2 N_{0} \frac{\partial \mathcal{F}_{S^{2}}}{\partial S_{0}}+N_{1} \frac{\partial \mathcal{F}_{S^{2}}}{\partial S_{1}}+4 \mathcal{F}_{R P^{2}} .
\end{aligned}
$$


where $\mathcal{F}_{S^{2}}$ and $\mathcal{F}_{R P^{2}}$ are the $S^{2}$ and $R P^{2}$ contributions, respectively, to the free energy of the associated $S O / S p(N)$ matrix model, as defined in Appendix A. The scales $\Lambda_{0}, \Lambda_{1}$ in (5.3) are the energy scales of the low energy $S O\left(2 N_{0}\right) / S p\left(N_{0}\right), U\left(N_{1}\right)$ theories with the $\Phi$ field integrated out, respectively. They are related to the high energy scale $\Lambda$ by the matching conditions as in (2.11) and (2.12), which yields

$$
\begin{aligned}
\left(\Lambda_{0}\right)^{3\left(N_{0} \mp 1\right)} & =m^{N_{0}-N_{1} \mp 1} g^{N_{1}} \Lambda^{2(N \mp 1)}, \\
\left(\Lambda_{1}\right)^{3 N_{1}} & =2^{-N_{1}} m^{-2 N_{0} \pm 2} g^{2 N_{0}+N_{1} \mp 2} \Lambda^{4(N \mp 1)} .
\end{aligned}
$$

The matrix model free energy is computed in Appendix A, and the result is

$$
\begin{aligned}
W_{\text {pert }}=\left(N_{0} \mp 1\right)\left[\left(\frac{3}{2} S_{0}^{2}-8 S_{0} S_{1}+2 S_{1}^{2}\right) \alpha+\left(-\frac{9}{2} S_{0}^{3}+42 S_{0}^{2} S_{1}-36 S_{0} S_{1}^{2}+4 S_{1}^{3}\right) \alpha^{2}\right. \\
\left.+\left(\frac{45}{2} S_{0}^{4}-\frac{932}{3} S_{0}^{3} S_{1}+523 S_{0}^{2} S_{1}^{2}-\frac{608}{3} S_{0} S_{1}^{3}+\frac{40}{3} S_{1}^{4}\right) \alpha^{3}\right] \\
+N_{1}\left[\left(-2 S_{0}^{2}+2 S_{0} S_{1}\right) \alpha+\left(7 S_{0}^{3}-18 S_{0}^{2} S_{1}+6 S_{0} S_{1}^{2}\right) \alpha^{2}\right. \\
\left.+\left(-\frac{233}{6} S_{0}^{4}+\frac{524}{3} S_{0}^{3} S_{1}-152 S_{0}^{2} S_{1}^{2}+\frac{80}{3} S_{0} S_{1}^{3}\right) \alpha^{3}\right]+\mathcal{O}\left(\alpha^{4}\right)
\end{aligned}
$$

where $\alpha \equiv g / m^{2}$. The result (5.5) agrees with the one obtained in [38], where the glueball superpotential was calculated by evaluating the periods (3.6). The full result, (5.3) and (5.4), has the expected general form (3.12):

$$
W_{\mathrm{eff}}=\left(N_{0} \mp 1\right) \Pi_{0}\left(S_{0}, S_{1}\right)+N_{1} \Pi_{1}\left(S_{0}, S_{1}\right)-2 \pi i \alpha\left(\frac{1}{2} S_{0}+S_{1}\right) .
$$

The general prescription in section 3 reads in the present case as follows:

\section{- $\mathrm{SO}(2 \mathrm{~N}) / \mathrm{Sp}(\mathrm{N}) \rightarrow \mathrm{SO}(2 \mathrm{~N}) / \mathrm{Sp}(\mathrm{N}) \times \mathrm{U}(0)$ (unbroken $\mathrm{SO} / \mathrm{Sp}$ ):}

Set $N_{1}=0, S_{1}=0$. Then the superpotential is

$$
W_{\text {eff }}\left(S_{0}, N_{0}\right)=\left(N_{0} \mp 1\right) S_{0}\left[1-\ln \left(S_{0} / \Lambda_{0}^{3}\right)\right]+\left.2\left(N_{0} \mp 1\right) \frac{\partial \mathcal{F}_{S^{2}}}{\partial S_{0}}\right|_{S_{1}=0} .
$$

This superpotential coincides with that of $U(2 N \mp 2)$ with adjoint and breaking pattern $U(2 N \pm 2) \rightarrow U(2 N \mp 2) \times U(0) \times U(0)$, as expected from the map (3.14) or (3.16). As shown in 21] for $S O(2 N)$, this matrix model result agrees with that of standard gauge theory, via using the corresponding Seiberg-Witten curve.

- $\mathrm{SO}(2 \mathrm{~N}) \rightarrow \mathrm{SO}(0) \times \mathrm{U}(\mathrm{N}):$

Set $N_{0}=0$ and take $S_{0} \rightarrow 0$, which eliminates the Veneziano-Yankielowicz part for the $S O(0)$. Then the superpotential is

$$
\begin{aligned}
W_{\text {eff }}\left(S_{1}\right) & =N S_{1}\left[1-\ln \left(S_{1} / \Lambda_{1}^{3}\right)\right]+\left.4 \mathcal{F}_{R P^{2}}\right|_{S_{0}=0} . \\
& =-\left.\Pi_{0}\left(S_{0}, S_{1}\right)\right|_{S_{0}=0}+\left.N \Pi_{1}\left(S_{0}, S_{1}\right)\right|_{S_{0}=0}-2 \pi i \alpha S_{1} .
\end{aligned}
$$


Note that $\left.\frac{\partial \mathcal{F}_{S^{2}}}{\partial S_{1}}\right|_{S_{0}=0}=0$ because $\mathcal{F}_{S^{2}}$ does not contain terms with $S_{1}$ only (all terms are of the form $S_{0}^{n} S_{1}^{m}$ with $n>0$ ). And though the Veneziano-Yankielowicz part of the superpotential for $S O(0)$ is eliminated via $S_{0} \rightarrow 0$, the -1 units of flux associated with $S O(0)$ does make a contribution in (5.8), with the non-zero terms in $-\left.\Pi_{0}\left(S_{0}, S_{1}\right)\right|_{S_{0}=0}$ in the second line of (5.8) coming from the term $\left.4 \mathcal{F}_{R P^{2}}\right|_{S_{0}=0}$.

- $\mathrm{SO}(2 \mathrm{~N}) \rightarrow \mathrm{SO}(2) \times \mathrm{U}(\mathrm{N}-1):$

Set $N_{0}=1, S_{0}=0$ and remove the Veneziano-Yankielowicz part for the $S O(2)$. Then the superpotential is

$$
W_{\mathrm{DV}}\left(S_{1}\right)=(N-1) S_{1}\left[1-\ln \left(S_{1} / \Lambda_{1}^{3}\right)\right]
$$

where we used $\left.\frac{\partial \mathcal{F}_{S^{2}}}{\partial S_{1}}\right|_{S_{0}=0}=0$ again. Integrating out $S_{1}$ gives

$$
W_{\text {low }}=(N-1) \Lambda_{1}^{3}=\frac{1}{2}(N-1) g \Lambda^{4}
$$

- $\mathbf{S p}(\mathrm{N}) \rightarrow \mathbf{S p}(0) \times \mathbf{U}(\mathrm{N}):$

Set $N_{0}=0$ in the equation and keep both $S_{0}$ and $S_{1}$. Then the superpotential is

$$
\begin{aligned}
W_{\mathrm{DV}}\left(S_{0}, N_{0}, S_{1}, N_{1}\right) & =S_{0}\left[1-\ln \left(S_{0} / \Lambda_{0}^{3}\right)\right]+N S_{1}\left[1-\ln \left(S_{1} / \Lambda_{1}^{3}\right)\right]+W_{\text {pert }} \\
W_{\text {pert }} & =N_{1} \frac{\partial \mathcal{F}_{S^{2}}}{\partial S_{1}}+4 \mathcal{F}_{R P^{2}}=N_{1} \frac{\partial \mathcal{F}_{S^{2}}}{\partial S_{1}}+2 \frac{\partial \mathcal{F}_{S^{2}}}{\partial S_{0}}
\end{aligned}
$$

For various breaking patterns, we integrated out the glueball superfield(s) from the glueball superpotential (5.7)-(5.11), and calculated the low energy superpotential $W_{\text {low }}$ as a function of coupling constants $m, g$, and the scale $\Lambda$. Having obtained the actual matrix model results, we can compare to the superpotential as computed via standard gauge theory methods, such as via factorizing of the Seiberg-Witten curve. This method

is reviewed in Appendix B, and the results are found to agree with the matrix model results completely. The resulting $W_{\text {low }}$ is shown in Table 1.

The $S O(2 N+1)$ theory with adjoint in the $S O(2 N+1) \rightarrow U(1)^{N}$ vacuum was studied diagrammatically in [39].

\section{2. $S p(N)$ theory with antisymmetric tensor}

Consider $S p(N)$ theory with an antisymmetric tensor chiral superfield $A=-A^{T}$. Take cubic tree level superpotential

$$
W_{\text {tree }}=\frac{1}{2} \operatorname{Tr}[W(\Phi)], \quad W(x)=\frac{m}{2} x^{2}+\frac{g}{3} x^{3}
$$




\begin{tabular}{|c|c|}
\hline breaking pattern & $W_{\text {low }}^{\prime}$ \\
\hline \hline$S O(4) \rightarrow S O(4) \times U(0)$ & $m \Lambda^{2}+\frac{3}{2} g \Lambda^{4}$ \\
$S O(4) \rightarrow S O(2) \times U(1)$ & $\frac{1}{2} g \Lambda^{4}$ \\
$S O(4) \rightarrow S O(0) \times U(2)$ & $m \Lambda^{2}-\frac{1}{2} g \Lambda^{4}$ \\
\hline$S O(6) \rightarrow S O(6) \times U(0)$ & $2 m \Lambda^{2}+3 g \Lambda^{4}$ \\
$S O(6) \rightarrow S O(4) \times U(1)$ & $g \Lambda^{4}$ \\
$S O(6) \rightarrow S O(2) \times U(2)$ & $g \Lambda^{4}$ \\
$S O(6) \rightarrow S O(0) \times U(3)$ & $\frac{3}{2}\left(m^{2} g \Lambda^{8}\right)^{1 / 3}-\frac{1}{2}\left(g^{5} \Lambda^{16} / m^{2}\right)^{1 / 3}-g^{3} \Lambda^{8} / 6 m^{2}+\cdots$ \\
\hline$S O(8) \rightarrow S O(8) \times U(0)$ & $3 m \Lambda^{2}+\frac{9}{2} g \Lambda^{4}$ \\
$S O(8) \rightarrow S O(6) \times U(1)$ & $2(m g)^{1 / 2} \Lambda^{3}+g^{2} \Lambda^{6} / m-\left(g^{7} / m^{5}\right)^{1 / 2} \Lambda^{9}+\cdots$ \\
$S O(8) \rightarrow S O(4) \times U(2)$ & $2 g^{2} \Lambda^{6} / m-4 g^{5} \Lambda^{12} / m^{4}+32 g^{8} \Lambda^{18} / m^{7}+\cdots$ \\
$S O(8) \rightarrow S O(2) \times U(3)$ & $\frac{3}{2} g \Lambda^{4}$ \\
$S O(8) \rightarrow S O(0) \times U(4)$ & $2(m g)^{1 / 2} \Lambda^{3}-g^{2} \Lambda^{6} / 2 m+\frac{1}{4}\left(g^{7} / m^{5}\right)^{1 / 2} \Lambda^{9}+\cdots$ \\
\hline \hline$S p(2) \rightarrow S p(2) \times U(0)$ & $2 m \Lambda^{2}+3 g \Lambda^{4}$ \\
$S p(2) \rightarrow S p(0) \times U(1)$ & $g \Lambda^{4}$ \\
\hline$S p(4) \rightarrow S p(4) \times U(0)$ & $3 m \Lambda^{2}+\frac{9}{2} g \Lambda^{4}$ \\
$S p(4) \rightarrow S p(2) \times U(1)$ & $2(m g)^{1 / 2} \Lambda^{3}+g^{2} \Lambda^{6} / m-\left(g^{7} / m^{5}\right)^{1 / 2} \Lambda^{9}+\cdots$ \\
$S p(4) \rightarrow S p(0) \times U(2)$ & $2 g^{2} \Lambda^{6} / m-4 g^{5} \Lambda^{12} / m^{4}+32 g^{8} \Lambda^{18} / m^{7}+\cdots$ \\
\hline
\end{tabular}

Table 1: The low energy superpotential calculated from the factorization of the Seiberg-Witten curve and from matrix model. In the above, the classical contribution has been subtracted: $W_{\text {low }}=-N_{1} m^{2} / 4 g+W_{\text {low }}^{\prime}$.

where $\Phi=A J$, and $J$ is the invariant antisymmetric tensor $J=\mathbf{1}_{N} \otimes i \sigma^{2}$. We do not require $A$ to be traceless, i.e. $\operatorname{Tr}[\Phi]=\operatorname{Tr}[A J] \neq 0$. By a complexified $S p(N)$ gauge rotation, $\Phi$ can be diagonalized as 40

$$
\Phi \cong \operatorname{diag}\left[\lambda_{1}, \cdots, \lambda_{N}\right] \otimes \mathbf{1}_{2}, \quad \lambda_{i} \in \mathbf{C} .
$$

The superpotential (5.12) has critical points at $\lambda=0,-m / g$. The classical supersymmetric vacuum of the theory is given by distributing $N_{1}$ and $N_{2}$ "eigenvalues" $\lambda_{i}$ at the critical point $\lambda=0$ and $\lambda=-m / g$, respectively, with $N_{1}+N_{2}=N$, breaking $S p(N) \rightarrow S p\left(N_{1}\right) \times$ $S p\left(N_{2}\right)$.

The glueball superpotential is calculated from the associated $S p(N)$ matrix model as

$$
\begin{gathered}
W_{\mathrm{DV}}\left(S_{1}, N_{1} ; S_{2}, N_{2}\right)=\left(N_{1}+1\right) S_{1}\left[1-\ln \left(S_{1} / \Lambda_{1}^{3}\right)\right] \\
\quad+\left(N_{2}+1\right) S_{2}\left[1-\ln \left(S_{2} / \Lambda_{2}^{3}\right)\right]+W_{\text {pert }} \\
W_{\text {pert }}=2 N_{1} \frac{\partial \mathcal{F}_{S^{2}}}{\partial S_{1}}+2 N_{2} \frac{\partial \mathcal{F}_{S^{2}}}{\partial S_{2}}+4 \mathcal{F}_{R P^{2}}
\end{gathered}
$$


The scales $\Lambda_{1}, \Lambda_{2}$ in (5.3) respectively are the energy scales of the low energy $S p\left(N_{1}\right)$, $S p\left(N_{2}\right)$ theories with the $\Phi$ field integrated out. They are related to the high energy scale $\Lambda$ by the matching conditions as in (2.14), which yields

$$
\begin{aligned}
& \left(\Lambda_{1}\right)^{3\left(N_{1}+1\right)}=m^{N_{1}-2 N_{2}-1} g^{2 N_{2}} \Lambda^{2 N+4}, \\
& \left(\Lambda_{2}\right)^{3\left(N_{2}+1\right)}=(-1)^{N_{2}-1} m^{-2 N_{1}+N_{2}-1} g^{2 N_{1}} \Lambda^{2 N+4} .
\end{aligned}
$$

The matrix model free energy is computed in Appendix A, and the result is

$$
\begin{aligned}
W_{\text {pert }}=2\left(N_{1}+1\right) & {\left[\left(-S_{1}^{2}+5 S_{1} S_{2}-\frac{5}{2} S_{2}^{2}\right) \alpha+\left(-\frac{16}{3} S_{1}^{3}+\frac{91}{2} S_{1}^{2} S_{2}-59 S_{1} S_{2}^{2}+\frac{91}{6} S_{2}^{3}\right) \alpha^{2}\right.} \\
& \left.+\left(-\frac{140}{3} S_{1}^{4}+\frac{1742}{3} S_{1}^{3} S_{2}-1318 S_{1}^{2} S_{2}^{2}+\frac{2636}{3} S_{1} S_{2}^{3}-\frac{871}{6} S_{2}^{4}\right) \alpha^{3}\right] \\
& +2\left(N_{2}+1\right)\left[S_{1} \leftrightarrow S_{2}, \alpha \rightarrow-\alpha\right],
\end{aligned}
$$

where $\alpha \equiv g^{2} / m^{3}$. This is as expected from the map (3.17) and [11].

In particular, let us concentrate on the unbroken case, $N_{2}=0$. Unlike [10,15], we do not set $S_{2}=0$, but rather keep $S_{2}$ non-zero and extremize with respect to it, according to our general prescription, to obtain the actual matrix model result. After integrating out $S_{1}$ and $S_{2}$ from (5.16), we obtain the superpotential as a power series in $\Lambda_{1}$ and $\Lambda_{2}$ as

$$
W_{\text {low }}=(N+1) \Lambda_{1}^{3}+\Lambda_{2}^{3}+\left(\text { higher order terms in } \Lambda_{1,2}\right) .
$$

The matching relation (5.15) gives

$$
\Lambda_{2}^{3}=-\left(\Lambda_{1}^{3}\right)^{N+1} \alpha^{N},
$$

so the terms containing $\Lambda_{2}$ in (5.17) starts to contribute to the superpotential at order $\left(\Lambda_{1}^{3}\right)^{N+1}$, i.e. like $S p(N)$ instantons. If we use the relation (5.18) and write out all the terms in (5.17), we obtain

$$
\begin{array}{ll}
N=0: & W_{\text {low }}=\mathcal{O}\left(\alpha^{4}\right), \\
N=1: & W_{\text {low }}=2 \Lambda_{1}^{3}+\mathcal{O}\left(\alpha^{4}\right), \\
N=2: & W_{\text {low }}=3 \Lambda_{1}^{3}-\Lambda_{1}^{6} \alpha-2 \Lambda_{1}^{9} \alpha^{2}-\frac{187}{27} \Lambda_{1}^{12} \alpha^{3}+\mathcal{O}\left(\alpha^{4}\right), \\
N=3: & W_{\text {low }}=4 \Lambda_{1}^{3}-3 \Lambda_{1}^{6} \alpha-\frac{47}{6} \Lambda_{1}^{9} \alpha^{2}-\frac{75}{2} \Lambda_{1}^{12} \alpha^{3}+\mathcal{O}\left(\alpha^{4}\right), \\
N=4: & W_{\text {low }}=5 \Lambda_{1}^{3}-5 \Lambda_{1}^{6} \alpha-13 \Lambda_{1}^{9} \alpha^{2}-65 \Lambda_{1}^{12} \alpha^{3}+\mathcal{O}\left(\alpha^{4}\right) .
\end{array}
$$

Thus properly accounting for $S_{2}$, it turns out that these matrix model results agree perfectly, up to the order presented, with the standard gauge theory results (Eq. (4.13) of 
[10]). (The discrepancies found in [10] set in at order $\Lambda_{1}^{3(N+1)}$, and are cancelled e.g. by (5.18).) In (5.19), for $N=0,1$, there were rather remarkable cancellations between the instanton contributions from $S p\left(N_{1}\right)$ and $S p\left(N_{2}\right)$. This will be further discussed and generalized in the next section.

The matrix model prediction for the superpotential of the $S O(2 N)$ theory with symmetric tensor can similarly be obtained by simply changing the $N_{i}+1$ in (5.16) to $N_{i}-1$. It should be possible to compute the superpotential from gauge theory using the duality for this theory [41]. The result is expected to be compatible with the map (3.20) to the superpotential computed for the $U(N-2 K)$ theory with adjoint.

\section{Residual Instantons: String theory (matrix model) versus gauge theory}

A remarkable aspect of the string theory (matrix model) computation of the effective superpotential is that (1.3) can be obtained purely in terms of the dynamics of the lowenergy $\prod_{i=1}^{K} G\left(N_{i}\right)$ theory on the RHS of (1.1). The only information needed about the high-energy $G(N)$ gauge theory is the perturbative contribution of the $G(N) / \prod_{i} G_{i}\left(N_{i}\right)$ ghosts to the glueball superpotential (1.3), as discussed in [42], along with the matching relations connecting the scales $\Lambda_{i}$ of the low-energy $G_{i}\left(N_{i}\right)$ factors to the scale $\Lambda$ of the highenergy $G(N)$ theory. This is very different from the conventional description of standard gauge theory, where there can be non-perturbative contributions to $W_{\text {low }}$ which are not readily seen in terms of the low-energy theory on the RHS of (1.1). An example of such an effect is instantons in the broken part of the group when $\pi_{3}\left(G(N) / \prod_{i} G_{i}\left(N_{i}\right)\right) \neq 0$ (see

e.g. 43]). Nevertheless, the string theory/ matrix model does properly reproduce such effects, via a low-energy description.

A gauge theory interpretation for the string theory/ matrix model results was given in [8,9]: the string theory / matrix model results actually refer to a particularly natural UV completion of the original $G(N)$ theory, where it is embedded in the supergroup $G(N+k \mid k)$ with $k$ large. This latter theory has a Higgs branch, where $k$ can be reduced successively, eventually Higgsing the theory down to the original $G(N)$ theory. More generally, the theory with breaking pattern (1.1) is replaced with

$$
G(N+k \mid k) \rightarrow \prod_{i=1}^{K} G_{i}\left(N_{i}+k_{i} \mid k_{i}\right)
$$

which has a Higgs branch flat direction connecting it to (1.1). Consideration of the particular matter content of the $G(N+k \mid k)$ theories along the Higgs branch, which often has extended supersymmetry, suggests that no dynamically generated superpotential ever lifts this Higgs branch moduli space, i.e. that the superpotentials of these particular theories are always independent of the location of the theory on this Higgs branch [9]. Moving along 
the Higgs branch has the effect of reducing $k$, and this expected independence of the superpotential of the position on the Higgs branch fits with the fact that the $G(N+k \mid k)$ matrix model results are $k$ independent, because all $k$ dependence cancels in the supertraces.

Because of the expected independence of the superpotential on the Higgs branch, and because we Higgs back to the original $G(N)$ theory, in most cases, this "F-completion" of the original $G(N)$ theory into the $G(N+k \mid k)$ theory is of no consequence. There are, however, a few rare exceptions, where the superpotential of the Higgsed $G(N+k \mid k)$ theory differs from that of the standard $G(N)$ theory. This difference comes from residual instantons in $G(N+k \mid k) / G(N)$, which need not decouple even if $G(N+k \mid k)$ is Higgsed to $G(N)$ far in the UV. As verified in [9], these residual instanton contributions precisely account for the few differences between the string theory (matrix model) results and standard gauge theory, for example the glueball superpotentials, with coefficient $h=1$, for $U(1)$ and $S p(0)$, e.g. with an adjoint and quadratic superpotential.

In many cases, however, these residual instanton contributions sum up to yield precisely the result expected from standard gauge theory, including superpotential contributions which in standard gauge theory would not have had a known low-energy description. In particular, residual instanton contributions which could have lead to potential discrepancies with standard gauge theory often completely cancel. The cancellation occurs once one sums over the different terms $i$ in (1.2), upon using the precise matching relation between the low-energy scales $\Lambda_{i}$, and the original high-energy scale $\Lambda$.

As an example, consider $U(K)$ with adjoint matter and breaking pattern $U(K) \rightarrow$ $U(1)^{K}$. For $K=1$ the string theory (matrix model) description includes a residual instanton effect, yielding $W_{\text {low }}=\Lambda_{L}^{3}$ rather than the standard gauge theory answer $W_{\text {sgt }}=0$ [9]. But for all $K>1$ the string theory/ matrix model result is $W_{\text {low }}=0$, in agreement with the standard gauge theory expectation for $U(K) \rightarrow U(1)^{K}$. The result $W_{\text {low }}=0$ looks like a remarkable cancellation because the glueball superpotential $W_{\text {eff }}\left(S_{1}, \ldots S_{K}\right)$ is quite non-trivial. Nevertheless, upon solving for the $\left\langle S_{i}\right\rangle$ and plugging back in, the exact result for $W_{\text {low }}=W_{\text {eff }}\left(\left\langle S_{i}\right\rangle\right)$ is zero, as was proven in [44].

To illustrate this cancellation, consider the leading order gaugino condensation contribution to $W_{\text {eff }}\left(S_{i}\right)$ in the string theory (matrix model) constructions, where the unbroken $U(1)$ factors in $U(K) \rightarrow U(1)^{K}$ contribute as in (2.15), with $h_{i}=1$, unlike in standard gauge theory:

$$
W_{\mathrm{gc}}\left(S_{i}\right)=\sum_{i=1}^{k} S_{i}\left(\log \left(\frac{\Lambda_{i}^{3}}{S_{i}}\right)+1\right)
$$

with $\Lambda_{i}^{3}=g_{K+1} \Lambda^{2 N} \prod_{j \neq i}\left(a_{j}-a_{i}\right)^{-1}$ by using $(2.10)$ with all $N_{i}=1$. Though this is a 
non-trivial superpotential, it vanishes upon integrating out the $S_{i}$ :

$$
W_{\mathrm{gc}}\left(\left\langle S_{i}\right\rangle\right)=\sum_{i=1}^{K} \Lambda_{i}^{3}=g_{K+1} \Lambda^{2 N} \sum_{i=1}^{K} \prod_{j \neq i}\left(a_{j}-a_{i}\right)^{-1}=g_{k+1}^{2} \Lambda^{2 N} \oint \frac{d x}{2 \pi i} \frac{1}{W^{\prime}(x)}=0
$$

The contour in (6.3) encloses all the zeros of $W^{\prime}(x)$, and we get zero for all $K>1$ by pulling the contour off to infinity. We see here why $K=1$ is different: we then get a residue at infinity, leading to the low energy superpotential $W_{U(1)}=\Lambda_{L}^{3}$, as in (2.9), with $h_{U(1)}=1$ as in (1.4).

To give another example of such a cancellation of residual instanton effects, consider the string theory (matrix model) result for $S p(N)$ with an antisymmetric tensor $A$, with $W_{\text {tree }}$ having $K$ critical points, for the case $N=0$. For the case of $K=1$, the superpotential is just a mass term for $A$ and the low-energy superpotential is the $S p(0)$ gaugino condensation superpotential, with $h(S p(0))=1: W=\Lambda^{3}$, unlike standard gauge theory. Again, this can be understood as a residual instanton effect in the F-completion of $S p(N)$ to $S p(N+k \mid k)$, which is present precisely for the case $N=0$ [9]. For a higher order superpotential, $K>1$, we would write the breaking pattern as $S p(0) \rightarrow S p(0)^{K}$. For all $K>1$, the residual instanton effects all cancel, precisely as in the $U(K) \rightarrow U(1)^{K}$ example discussed above; in fact, the two theories have the same effective superpotential $W_{\text {eff }}$ (aside from the classical difference), as discussed in [11] and sect. 3.3. Thus, for example, (6.3) can also be interpreted as the leading gaugino condensation contributions from the $S p(0)^{K}$ factors, and where we now use the matching relation (2.14) to relate the $\Lambda_{i}^{3}$ to $g_{K+1} \Lambda^{2 K} \prod_{j \neq i}\left(a_{j}-a_{i}\right)^{-1}$. Again, there is complete cancellation in $W_{\text {eff }}$ here, except for the case $K=1$.

More generally, for $S p(N)$ with antisymmetric, breaking as $S p(N) \rightarrow \prod_{i=1}^{K} S p\left(N_{i}\right)$, the results obtained via the string theory / matrix model glueball potential $W_{\text {eff }}\left(S_{1}, \ldots S_{K}\right)$, upon integrating out the $S_{i}$, appears to always agree with standard gauge theory results for the superpotential [40], as seen in the examples of [11] and (5.19). This agreement comes about via a remarkable interplay between the different terms $i$ in (3.12). If we treated the scales $\Lambda_{i}$ of the $S p\left(N_{i}\right)$ factors as if they were initially independent, each term $\hat{N}_{i} \Pi\left(\left\langle S_{i}\right\rangle\right)-2 \pi i \eta_{i}\left\langle S_{i}\right\rangle$ in (3.12) would be a complicated function of $\Lambda_{i}$, which does not have a known, conventional, interpretation in terms of standard gauge theory for the low-energy $S p\left(N_{i}\right)$ factor. But upon adding the different $i$ terms and using the matching relations relating $\Lambda_{i}$ to $\Lambda$, e.g. (2.14), one nevertheless obtains the standard gauge theory results, thanks to an intricate interplay between the different terms $i$.

By the map of (3.17) [1], the agreement between string theory / matrix models and standard gauge theory for $S p(N)$ with antisymmetric can be phrased as such an agreement for $U(N+K)$ with adjoint and breaking pattern $U(N+K) \rightarrow \prod_{i=1}^{K} U\left(N_{i}+1\right)$. 
As another example, consider $U(N)$ with adjoint $\Phi$ and superpotential having $K=$ $N-1$, in the vacuum where $U(N) \rightarrow U(2) \times U(1)^{N-2}$. Factorizing the Seiberg-Witten curve yields for the exact superpotential [46]

$$
W_{\text {exact }}=W_{\mathrm{cl}}(g) \pm 2 g_{N} \Lambda^{N} .
$$

The map of [11] and sect. 3.3 relates this to $S p(1) \rightarrow S p(1) \times S p(0)^{N-2}$, where the exact gauge theory result agrees with (6.4), up to the classical shift, upon using the relation (3.18). A priori, one might expect the string theory / matrix model result to disagree with (6.4), due to residual instanton contributions from the $U(1)^{N-2}$ or the $S p(0)^{N-2}$ in $U(N) \rightarrow U(2) \times U(1)^{N-2}$ and $S p(1) \rightarrow S p(1) \times S p(0)^{N-2}$ respectively. But the string theory / matrix model result nevertheless agrees with (6.4), thanks to the interplay between the different terms. Consider, in particular, the case $U(3) \rightarrow U(2) \times U(1)$. The fact that (6.4) will only hold if remarkable cancellations occur upon integrating out $S_{1}$ and $S_{2}$ from the non-trivial $W\left(S_{1}, S_{2}\right)$, was discussed in [3], where the cancellations were verified to indeed occur, up to order $\alpha^{3}$. This is checked to one higher order in (5.19), since it is related to $S p(1) \rightarrow S p(1) \times S p(0)$ by the map of [11] and sect. 3.3. The leading order cancellation, say in terms of $U(3) \rightarrow U(2) \times U(1)$, is between $U(1)$ gaugino condensation, $\Lambda_{2}^{3}$, and a higher order term coming from integrating out $S_{1}$ from $W_{\text {pert }}\left(S_{i}\right)$.

The residual instanton contributions associated with the UV completion (6.1), as opposed to the standard gauge theory results for (1.1) do not always cancel, however. The cases where we find non-cancellations are when the degree of the superpotential is sufficiently large, so that it contains terms which are not independent moduli. As an example, consider $U(1)$ with $W_{\text {tree }}$ as in (2.2) having $K$ minima, breaking $U(1) \rightarrow U(1) \times$ $U(0)^{K-1}$. The gaugino condensation contribution to the superpotential, according to the string theory (matrix model) construction, is given by (2.15) with $h_{1}=1$ and all other $h_{i}=0$ and their $S_{i}$ set to zero. Upon integrating out $S_{1}$, we thus obtain the superpotential

$$
W_{\mathrm{gc}}=\Lambda_{1}^{3}=\Lambda^{2} W^{\prime \prime}\left(a_{1}\right)=g_{K+1} \Lambda^{2} \prod_{j \neq 1}\left(a_{j}-a_{1}\right)
$$

where we used the matching relation (2.10) with $N_{1}=1$ and all other $N_{j}=0$.

The full low-energy effective superpotential $W_{\text {low }}\left(g_{i}, \Lambda\right)$ can be regarded as the generating function for the operator expectation values:

$$
\left\langle u_{j}\right\rangle=\frac{\partial W_{\mathrm{low}}\left(g_{i}, \Lambda\right)}{\partial g_{j}} \quad u_{i} \equiv \frac{1}{j} \operatorname{Tr} \Phi^{j} .
$$

In the $U(1)$ theory, we have classical relations $u_{j}=\frac{1}{j} u_{1}^{j}$. But the quantum contribution (6.5) (along with additional, higher order contributions) imply quantum deformation of 
these classical relations, due to the residual instanton effects in the $U(1+k \mid k) \rightarrow U(1+$ $\left.k_{1} \mid k_{1}\right) \times U\left(k_{2} \mid k_{2}\right) \ldots U\left(k_{K} \mid k_{K}\right)$ F-completion. For the simplest such example, consider $U(1)$ with $W_{\text {tree }}=\frac{1}{2} m \Phi^{2}+\lambda \Phi$. The low-energy superpotential is

$$
W_{\text {low }}=-\frac{\lambda^{2}}{2 m}+m \Lambda^{2}
$$

with the first term the classical contribution and the second the residual instanton. Using (6.6) we then get

$$
\left\langle u_{1}\right\rangle=-\frac{\lambda}{m}, \quad,\left\langle u_{2}\right\rangle=\frac{\lambda^{2}}{2 m^{2}}+\Lambda^{2} \quad \text { i.e. } \quad\left\langle u_{2}\right\rangle=\frac{1}{2}\left\langle u_{1}^{2}\right\rangle+\Lambda^{2},
$$

which can be regarded as an instanton correction to the composite operator $u_{2}$.

As another such example, consider $U(2)$ with an adjoint and $W_{\text {tree }}$ having $K$ minima, in the vacuum where the gauge group is broken as $U(2) \rightarrow U(1) \times U(1) \times U(0)^{K-2}$. The gaugino condensation contribution to $W_{\text {low }}$ is

$$
W_{\mathrm{gc}}=\Lambda_{1}^{3}+\Lambda_{2}^{3}=g_{K+1} \Lambda^{4}\left(\frac{\prod_{j=3}^{K}\left(a_{j}-a_{1}\right)-\prod_{j=3}^{K}\left(a_{j}-a_{2}\right)}{a_{2}-a_{1}}\right) .
$$

For example, for $U(2)$ with $W_{\text {tree }}$ having $K=3$ critical points, we break $U(2) \rightarrow U(1) \times$ $U(1) \times U(0)$ and $(6.9)$ leads to

$$
W_{\mathrm{gc}}=g_{4} \Lambda^{4} .
$$

Computing expectation values as in (6.6) this leads to

$$
\left\langle u_{4}\right\rangle=\left\langle u_{4}\right\rangle_{\mathrm{cl}}+\Lambda^{4}
$$

which can be interpreted as an instanton contribution to the composite operator $u_{4}=\operatorname{Tr} \Phi^{4}$ in $U(2)$ gauge theory. More generally, for $U(N)$ gauge theory, the independent basis of operators $u_{j}=\frac{1}{j} \operatorname{Tr} \Phi^{j}$ are only those with $j \leq N$, those with $j>N$ can be expressed as products of these basis operators via classical relations. But these relations can be affected by instantons. In particular, for $U(N)$ with an adjoint, the instanton factor is $\Lambda^{2 N}$, so operators $u_{j}$ with $j \geq 2 N$ can be affected. The above residual instanton contributions of the $U(N+k \mid k)$ UV completion can be interpreted as implying specific such instanton corrections to the higher Casimirs $u_{j}$.

A similar situation arises in the $\mathcal{N}=1^{*} U(N)$ theory, where the effective superpotential of the matrix model and conventional gauge theory differ by a contribution $N^{2} m^{3} E_{2}(N \tau)$ [17]; this was interpreted in [17] as differing operator definitions of $\operatorname{Tr} \Phi^{2}$ between gauge theory and the matrix model at the level of instantons. Related issues for multi-trace operators were seen in [18]. 


\section{Conclusions}

To compute the correct string theory / matrix model results, we should include or not include the glueball fields $S_{i}$ according to the prescription of this paper. Upon doing so, in all examples that we know of, the string theory / matrix model results agree with the results of standard gauge theory, at least in those cases where the relevant gauge theory does not suffer from UV ambiguities. In the case where such ambiguities are present, for example in defining composite operators appearing in $W_{\text {tree }}$, the string theory / matrix model results correspond to a particular UV definition of the theory. The agreement with standard gauge theory results is often due to a remarkable interplay between the different low-energy terms, found upon integrating out the glueball fields $S_{i}$, and connecting their scales $\Lambda_{i}$ via the appropriate matching relation to the scale $\Lambda$ of the original theory. In some cases, this interplay leads to complete cancellations of the residual instanton contributions to $W_{\text {low }}$ coming from the $G(N+k \mid k)$ completion [8,9]. Perhaps there is some additional structure governing the glueball superpotentials, which would make these remarkable cancellations more manifest.

\section{Acknowledgments}

We would like to thank M. Aganagic, F. Cachazo, S. Naculich and H. Schnitzer for valuable discussions.

K.I. was supported in part by DOE-FG03-97ER40546 and KI would also like to thank the Rutgers theory group for their support and hospitality while he was a visitor, where part of this work was done. P.K. was supported in part by NSF grant PHY-0099570. The research of A.V.R was supported by NSF grant 99-73935. A.V.R. and C.V. thank the 2003 Simons Workshop in Mathematics and Physics for a stimulating environment. C.V. would like to thank the hospitality of theory group at Caltech, where he was a Distinguished Gordon Moore Scholar. The research of C.V. was supported in part by NSF grants PHY9802709 and DMS-0074329.

\section{Appendix A. Matrix model calculation of superpotential}

In this appendix, after giving a proof for a general relation that relates $S^{2}$ and $R P^{2}$ contributions to the $S O / S p$ matrix model free energy, we compute explicitly the free energy of the matrix models associated with $S O / S p$ gauge theory with adjoint and $S p$ gauge theory with antisymmetric tensor. These matrix model results are used in section 5 to evaluate the glueball superpotential of the corresponding gauge theories. 


\section{A.1. Proof for relation between $\mathcal{F}_{S^{2}}$ and $\mathcal{F}_{R P^{2}}$}

Here we prove a general relation between the $S^{2}$ and $R P^{2}$ contributions to the $S O(2 N) / S p(N)$ matrix model free energy:

$$
\mathcal{F}_{R P^{2}}= \begin{cases}\mp \frac{1}{2} \frac{\partial \mathcal{F}_{S^{2}}}{\partial S_{0}} & S O(2 N) / S p(N) \text { with adjoint } \\ \mp \frac{1}{2} \sum_{i=1}^{K} \frac{\partial \mathcal{F}_{S^{2}}}{\partial S_{i}} & S O(2 N) / S p(N) \text { with symmetric/antisymmetric tensor. }\end{cases}
$$

The first equation was conjectured in [19,20] based on explicit diagrammatic calculations, and proven in [21] for the case of unbroken vacua. Here we will give a general matrix model proof for arbitrary breaking pattern. These relations are equivalent to the maps (3.14), (3.16), (3.17), and (3.20), which we obtained in sect. 3.3 immediately from the string theory geometric transition construction, accounting for the orientifold contributions to the fluxes.

Consider $U(\mathbf{N})$ and $S O(2 \mathbf{N}) / S p(\mathbf{N})$ matrix models which correspond to $U(N)$ and $S O(2 N) / S p(N)$ gauge theories with a two-index tensor matter field. The partition function is

$$
\mathbf{Z}=e^{-\frac{1}{\mathbf{g}^{2}} \mathbf{F}\left(\mathbf{S}_{i}\right)}=\int d \boldsymbol{\Phi} e^{-\frac{1}{\mathbf{g}} W_{\text {tree }}(\boldsymbol{\Phi})} .
$$

We denote matrix model quantities by boldface letters, following the notation of [15]. $\boldsymbol{\Phi}$ is an $\mathbf{N} \times \mathbf{N}$ (for $U(\mathbf{N})$ theory) or $2 \mathbf{N} \times 2 \mathbf{N}$ (for $S O(2 \mathbf{N}) / S p(\mathbf{N})$ theory) matrix corresponding to the $\Phi$ field in gauge theory, and the "action" $W_{\text {tree }}$ is defined in (2.2), (2.4) and (2.5). The matrix integral (A.2) is evaluated perturbatively around the general broken vacua of (2.1), with $N_{i}$ replaced by $\mathbf{N}_{i}$. We take the double scaling limit $\mathbf{N}_{i} \rightarrow \infty, \mathbf{g} \rightarrow 0$ with $\mathbf{g N}_{i} \equiv \mathbf{S}_{i}$ (for $U\left(\mathbf{N}_{i}\right)$ factors) or $2 \mathbf{g} \mathbf{N}_{i} \equiv \mathbf{S}_{i}$ (for $S O\left(2 \mathbf{N}_{i}\right) / S p\left(\mathbf{N}_{i}\right)$ factors) kept finite. The dependence of the free energy $\mathbf{F}\left(\mathbf{S}_{i}\right)$ on $\mathbf{N}_{i}$ are eliminated in favor of $\mathbf{S}_{i}$, and $\mathbf{F}\left(\mathbf{S}_{i}\right)$ is expanded in the 't Hooft expansion as

$$
\mathbf{F}\left(\mathbf{S}_{i}\right)=\sum_{\mathcal{M}} \mathbf{g}^{2-\chi(\mathcal{M})} \mathcal{F}_{\mathcal{M}}\left(\mathbf{S}_{i}\right)=\mathcal{F}_{S^{2}}+\mathbf{g} \mathcal{F}_{R P^{2}}+\cdots
$$

where the sum is over all compact topologies $\mathcal{M}$ of the matrix model diagrams written in the 't Hooft double-line notation, and $\chi(\mathcal{M})$ is the Euler number of $\mathcal{M}$.

The matrix model resolvent is defined as follows:

$$
\mathbf{R}(z) \equiv \mathbf{g}\left\langle\operatorname{Tr}\left[\frac{1}{z-\mathbf{\Phi}}\right]\right\rangle=\mathbf{R}_{S^{2}}(z)+\mathbf{g R}_{R P^{2}}(z)+\cdots
$$


For $U(N)$ theory with adjoint, the expansion parameter is $\mathbf{g}^{2}$ instead of $\mathbf{g}$, and in particular, $\mathbf{R}_{R P^{2}}(z) \equiv 0$. The resolvent and the free energy are related as

$$
\mathbf{R}_{\mathcal{M}}(z)=\frac{S}{z} \delta_{\chi(\mathcal{M}), 2}+\frac{1}{z^{2}} \frac{\partial \mathcal{F}_{\mathcal{M}}}{\partial g_{1}}+\frac{2}{z^{3}} \frac{\partial \mathcal{F}_{\mathcal{M}}}{\partial g_{2}}+\frac{3}{z^{4}} \frac{\partial \mathcal{F}_{\mathcal{M}}}{\partial g_{3}}+\cdots
$$

where $S=\sum_{i=1}^{K} S_{i}$. The resolvents can be determined uniquely by solving the matrix model loop equations (the loop equations for the relevant matrix models are summarized in [15]), under the condition

$$
\oint_{A_{i}} \frac{d z}{2 \pi i} \mathbf{R}_{S^{2}}(z)=\mathbf{S}_{i}, \quad \oint_{A_{i}} \frac{d z}{2 \pi i} \mathbf{R}_{R P^{2}}(z)=0
$$

$A_{i}$ is the contour around the $i$-th critical point of $W(z)$. In general, $\mathbf{R}(z)$ develops a cut around each critical point in the large $\mathbf{N}_{i}$ limit, and $A_{i}$ is taken to encircle the $i$-th cut. Note that the expression (A.5) should be understood as a Laurent expansion around $z=\infty$, and converges only if $|z|$ is larger than $r$ such that all the singularities (cuts) of the resolvent are inside the circle $C:|z|=r$.

On the other hand, gauge theory resolvents $R(z), T(z)$ (see e.g. [7]) are determined uniquely by solving the Konishi anomaly equations (the Konishi anomaly equations for the relevant gauge theories are summarized in [15]), under the condition

$$
\oint_{A_{i}} \frac{d z}{2 \pi i} R(z)=S_{i}, \quad \oint_{A_{i}} \frac{d z}{2 \pi i} T(z)=\left\{\begin{array}{ll}
N_{i} & U\left(N_{i}\right) \\
2 N_{i} & S O\left(2 N_{i}\right) / S p\left(N_{i}\right)
\end{array} .\right.
$$

As was shown in [15], the matrix model resolvents $\mathbf{R}_{S^{2}}(z), \mathbf{R}_{R P^{2}}(z)$ are related to the gauge theory resolvents $R(z), T(z)$ as

$$
R(z)=\mathbf{R}_{S^{2}}(z), \quad T(z)=\sum_{U\left(N_{i}\right)} N_{i} \frac{\partial}{\partial S_{i}} \mathbf{R}_{S^{2}}(z)+\sum_{\substack{S O\left(2 N_{i}\right) \\ / S p\left(N_{i}\right)}} 2 N_{i} \frac{\partial}{\partial S_{i}} \mathbf{R}_{S^{2}}(z)+4 \mathbf{R}_{R P^{2}}(z)
$$

with $S_{i}$ and $\mathbf{S}_{i}$ identified国.

First, consider $S O(2 N) / S p(N)$ theory with adjoint. The general breaking pattern is $S O(2 N) \rightarrow S O\left(2 N_{0}\right) \times U\left(N_{1}\right) \times \cdots \times U\left(N_{K}\right)$ or $S p(N) \rightarrow S p\left(N_{0}\right) \times U\left(N_{1}\right) \times \cdots \times U\left(N_{K}\right)$

5 The relation (A.8) is an obvious generalization of the formula in 15], which was for unbroken vacua, to an arbitrary breaking pattern. The gauge theory resolvents $R(z), T(z)$ given in (A.8) clearly satisfy the condition (A.7) provided that the matrix model resolvents $\mathbf{R}_{S^{2}}(z), \mathbf{R}_{R P^{2}}(z)$ satisfy the condition (A.6). 
(Eq. (2.1)), where $N=N_{0}+\sum_{i=1}^{K} N_{i}$. Note that the eigenvalues are distributed in a symmetric manner under $z \leftrightarrow-z$, and hence (A.7) is

$$
\begin{array}{llrl}
\oint_{A_{0}} \frac{d z}{2 \pi i} R(z) & =S_{0}, & \oint_{A_{i}} \frac{d z}{2 \pi i} R(z) & =\oint_{A_{-i}} \frac{d z}{2 \pi i} R(z)=S_{i} \\
\oint_{A_{0}} \frac{d z}{2 \pi i} T(z) & =2 N_{0}, & \oint_{A_{i}} \frac{d z}{2 \pi i} T(z) & =\oint_{A_{-i}} \frac{d z}{2 \pi i} T(z)=N_{i}
\end{array}
$$

where $i=1, \ldots, K$. The contours $A_{i}$ and $A_{-i}$ encircle counterclockwise the cuts around $z=a_{i}$ and $z=-a_{i}$, respectively. The relation (A.8) holds as it is, with the summation understood as over $S O\left(2 N_{0}\right) / S p\left(N_{0}\right)$ and $U\left(N_{i}\right), i=1, \ldots, K$.

It was shown in [13] that the resolvents of this $S O(2 N) / S p(N)$ theory are related to the resolvents $\tilde{R}(z)$ and $\tilde{T}(z)$ of $U(\tilde{N} \equiv 2 N \mp 2)$ theory with adjoint as follows:

$$
R(z)=\tilde{R}(z), \quad T(z)=\tilde{T}(z) \pm \frac{2}{z}
$$

The tree level superpotential of the $U(\tilde{N})$ theory is related to the one for the $S O(2 N) / S p(N)$ theory as $W^{U}(z)=W^{S O / S p}(z)$ (see (2.2) and (2.5)), and the breaking pattern is $U(\tilde{N}) \rightarrow U\left(N_{-K}\right) \times \cdots \times U\left(N_{-1}\right) \times U\left(2 N_{0} \mp 2\right) \times U\left(N_{1}\right) \times \cdots \times U\left(N_{K}\right)$ with $N_{-i}=N_{i}$, $1 \leq i \leq K$. Note that since there is no $z \leftrightarrow-z$ symmetry in the $U(\tilde{N})$ theory, the $U\left(N_{-i}\right)$ factors that are "images" for $S O(2 N) / S p(N)$ are "real" for $U(\tilde{N})$. In addition, the glueball $\tilde{S}$ of the $U(\tilde{N})$ theory is related to the glueball $S$ of the $S O(2 N) / S p(N)$ theory as $\tilde{S}_{0}=S_{0}, \tilde{S}_{i}=\tilde{S}_{-i}=S_{i}, 1 \leq i \leq K$. Therefore, e.g. the first equation in (A.10) is more precisely

$$
R\left(z, S_{j}\right)=\left.\tilde{R}\left(z, \tilde{S}_{j}\right)\right|_{\tilde{S}_{0}=S_{0}, \tilde{S}_{i}=\tilde{S}_{-i}=S_{i}} .
$$

Differentiating (A.11) with respect to $S_{j}$, we obtain

$$
\frac{\partial R}{\partial S_{0}}=\left.\frac{\partial \tilde{R}}{\partial \tilde{S}_{0}}\right|_{\substack{\tilde{S}_{0}=S_{0}, \tilde{S}_{i}=\tilde{S}_{-i}=S_{i}}} \quad, \quad \frac{\partial R}{\partial S_{j}}=\left.\left(\frac{\partial \tilde{R}}{\partial \tilde{S}_{j}}+\frac{\partial \tilde{R}}{\partial \tilde{S}_{-j}}\right)\right|_{\substack{\tilde{S}_{0}=S_{0}, \tilde{S}_{i}=\tilde{S}_{-i}=S_{i}}}
$$

where $1 \leq j \leq K$.

Now, using (A.8), let us translate the relation (A.10) among gauge theory resolvents into a relation among matrix model resolvents:

$$
\begin{aligned}
\mathbf{R}_{S^{2}} & =\tilde{\mathbf{R}}_{S^{2}}, \\
2 N_{0} \frac{\partial \mathbf{R}_{S^{2}}}{\partial S_{0}}+\sum_{i=1}^{K} N_{i} \frac{\partial \mathbf{R}_{S^{2}}}{\partial S_{i}}+4 \mathbf{R}_{R P^{2}} & =\left(2 N_{0} \mp 2\right) \frac{\partial \tilde{\mathbf{R}}_{S^{2}}}{\partial S_{0}}+\sum_{i=1}^{K} N_{i}\left(\frac{\partial \tilde{\mathbf{R}}_{S^{2}}}{\partial \tilde{S}_{i}}+\frac{\partial \tilde{\mathbf{R}}_{S^{2}}}{\partial \tilde{S}_{-i}}\right) \pm \frac{2}{z}
\end{aligned}
$$


Here $\tilde{\mathbf{R}}_{S^{2}}$ is the matrix model resolvent associated with the $U(\tilde{N})$ theory. Using (A.12) and the relations $\mathbf{R}_{S^{2}}=R, \tilde{\mathbf{R}}_{S^{2}}=\tilde{R}$ (Eq. (A.8)), we obtain

$$
\mathbf{R}_{R P^{2}}(z)=\mp \frac{1}{2} \frac{\partial}{\partial S_{0}} \mathbf{R}_{S^{2}}(z) \pm \frac{1}{2 z} .
$$

By expanding the resolvents around $z=\infty$ using (A.5) and comparing the coefficients, we obtain a relation between matrix model free energies:

$$
j \frac{\partial \mathcal{F}_{R P^{2}}}{\partial g_{j}}= \pm \frac{1}{2} \frac{\partial}{\partial S_{0}}\left(S \delta_{j 0}+j \frac{\partial \mathcal{F}_{S^{2}}}{\partial g_{j}}\right) \mp \frac{1}{2} \delta_{j 0} .
$$

where $j=0,2, \cdots, 2(K+1)$. The $j=0$ case is trivially satisfied since $S=S_{0}+2 \sum_{i=1}^{K} S_{i}$ here, while the $j=2,4, \ldots, 2(K+1)$ cases lead to the first equation of (A.1), which we wanted to prove.

Next, consider $S O(2 N) / S p(N)$ theory with symmetric/antisymmetric tensor. The breaking pattern is $S O(2 N) \rightarrow \prod_{i=1}^{K} S O\left(2 N_{i}\right)$ or $S p(N) \rightarrow \prod_{i=1}^{K} S p\left(N_{i}\right)$ (Eq. (2.1)), where $N=\sum_{i=1}^{K} N_{i}$. It was shown in [11] that the resolvents of this $S O / S p$ theory is related to the resolvents $\tilde{R}(z)$ and $\tilde{T}(z)$ of $U(\tilde{N} \equiv 2 N \mp 2 K)$ theory with adjoint as follows:

$$
R(z)=\tilde{R}(z), \quad T(z)=\tilde{T}(z) \pm \frac{d}{d z} \ln \left[W^{\prime}(z)^{2}+f_{K-1}(z)\right] .
$$

The tree level superpotential of the $U(\tilde{N})$ theory is related to the one for the $S O(2 N) / S p(N)$ theory as $W^{U}(z)=W^{S O / S p}(z)$ (see (2.2) and (2.4)), and the breaking pattern is $U(\tilde{N}) \rightarrow \prod_{i=1}^{K} U\left(2 N_{i} \mp 2\right)$. The glueball $S_{i}$ of the $U(\tilde{N})$ theory is taken to be the same as the glueball of the $S O(2 N) / S p(N)$ theory. In (A.16), $f_{K-1}(z)$ is a polynomial of degree $K-1$. Using (A.8), we can translate the relation (A.16) among gauge theory resolvents into a relation among matrix model resolvents:

$$
\mathbf{R}_{R P^{2}}(z)=\mp \frac{1}{2} \sum_{i=1}^{K} \frac{\partial}{\partial S_{i}} \mathbf{R}_{S^{2}}(z) \pm \frac{1}{4} \frac{d}{d z} \ln \left[W^{\prime}(z)^{2}+f_{K-1}(z)\right] .
$$

In order to extract the relation between matrix model free energies, let us multiply (A.17) by $z^{j}(0 \leq j \leq K+1)$ and integrate over $z$ along the contour $C$, introduced under (A.6), which encloses all the cuts around the critical points of $W(z)$. Taking

$$
W^{\prime}(z)^{2}+f_{K-1}(z)=g_{K+1}^{2} \prod_{i=1}^{K}\left(z-a_{i}^{+}\right)\left(z-a_{i}^{-}\right)
$$


the branching points of the cuts are at $z=a_{i}^{ \pm}$. The second term on the right hand side in (A.17) does not contribute to the contour integral unless $j=0$ :

$$
\begin{aligned}
& \mp \frac{1}{4} \oint_{C} \frac{d z}{2 \pi i} \sum_{i=1}^{K} z^{j}\left(\frac{1}{z-a_{i}^{+}}+\frac{1}{z-a_{i}^{-}}\right) \\
& \quad= \pm \frac{1}{4} \oint_{C} \frac{d w}{2 \pi i} \sum_{i=1}^{K} \frac{1}{w^{j+1}}\left(\frac{1}{1-a_{i}^{+} w}+\frac{1}{1-a_{i}^{-} w}\right)=\mp \frac{K}{2} \delta_{j 0}
\end{aligned}
$$

where $w=1 / z$, because all the poles $w=1 / a_{i}^{ \pm}$are outside of the contour $C$ (on the $w$-plane). On the contour $C$, we can use the Laurent expansion (A.5) to evaluate the contribution from the other terms, and the final result is

$$
j \frac{\partial \mathcal{F}_{R P^{2}}}{\partial g_{j}}= \pm \frac{1}{2} \sum_{i=1}^{K} \frac{\partial}{\partial S_{i}}\left(S \delta_{j 0}+j \frac{\partial \mathcal{F}_{S^{2}}}{\partial g_{j}}\right) \mp \frac{K}{2} \delta_{j 0}
$$

The $j=0$ case is trivially satisfied, while the $1 \leq j \leq K+1$ cases lead to the second equation of (A.1), which we wanted to prove.

\section{A.2. Computation of matrix model free energy: $S O(2 N) / S p(N)$ theory with adjoint}

Let us consider $S O(2 \mathrm{~N})$ matrix model which corresponds to $S O(2 N)$ gauge theory with adjoint. The tree level superpotential is taken to be quartic (5.1). The matrix variable $\boldsymbol{\Phi}$ in (A.2) is a real antisymmetric matrix and can be skew-diagonalized as

$$
\mathbf{\Phi} \cong \operatorname{diag}\left[\lambda_{1}, \cdots, \lambda_{\mathbf{N}}\right] \otimes i \sigma^{2}
$$

By changing the integration variables from $\boldsymbol{\Phi}$ to $\lambda_{i}$, we obtain

$$
\mathbf{Z} \sim \int \prod_{i=1}^{\mathbf{N}} d \lambda_{i} \prod_{i<j}^{\mathbf{N}}\left(\lambda_{i}^{2}-\lambda_{j}^{2}\right)^{2} e^{-\frac{1}{\mathrm{~g}} \sum_{i=1}^{\mathbf{N}}\left(-\frac{m}{2} \lambda_{i}^{2}+\frac{g}{4} \lambda_{i}^{4}\right)}
$$

where $\prod_{i<j}^{\mathbf{N}}\left(\lambda_{i}^{2}-\lambda_{j}^{2}\right)^{2}$ is the Jacobian for this change of variables 47,20. The polynomial $-\frac{m}{2} \lambda^{2}+\frac{g}{4} \lambda^{4}$ has critical points at $\lambda=0, \pm \sqrt{m / g}$, around which we would like to do perturbative expansion. For this purpose, we separate $\lambda$ 's into two groups as

$$
\lambda_{i}= \begin{cases}\lambda_{i_{0}}^{(0)} & i_{0}=1, \ldots, \mathbf{N}_{0} \\ \sqrt{m / g}+\lambda_{i_{1}}^{(1)} & i_{1}=1, \ldots, \mathbf{N}_{1}\end{cases}
$$

with $\mathbf{N}_{0}+\mathbf{N}_{1}=\mathbf{N}$, corresponding to the classical supersymmetric vacuum with breaking pattern $S O(2 \mathbf{N}) \rightarrow S O\left(2 \mathbf{N}_{0}\right) \times U\left(\mathbf{N}_{1}\right)$. We would like to evaluate the matrix integral 
(A.22) perturbatively around $\lambda^{(0,1)}=0$. If we expand the matrix model free energy in the coupling constant $g$ as

$$
\mathbf{F}=g f_{1}\left(\mathbf{N}_{0}, \mathbf{N}_{1}\right)+g^{2} f_{2}\left(\mathbf{N}_{0}, \mathbf{N}_{1}\right)+\cdots
$$

the loop expansion tells us that $f_{n}\left(\mathbf{N}_{0}, \mathbf{N}_{1}\right)$ is a polynomial of degree $n+2$. Therefore, by performing the matrix integral by computer for small values of $\mathbf{N}_{0}$ and $\mathbf{N}_{1}$, one can determine the polynomial $f_{n}$. If we rewrite $\mathbf{N}_{0,1}$ in favor of $S_{0}=2 \mathbf{g} \mathbf{N}_{0}$ and $S_{1}=\mathbf{g N} \mathbf{N}_{1}$, the expansion (A.24) arranges itself into the 't Hooft expansion (A.3), from which one can read off $\mathcal{F}_{S^{2}}, \mathcal{F}_{R P^{2}}$, etc.

Following the procedure sketched above, we computed the matrix model free energy as

$$
\begin{aligned}
\mathcal{F}_{S^{2}}= & \left(\frac{1}{4} S_{0}^{3}-2 S_{0}^{2} S_{1}+S_{0} S_{1}^{2}\right) \alpha+\left(-\frac{9}{16} S_{0}^{4}+7 S_{0}^{3} S_{1}-9 S_{0}^{2} S_{1}^{2}+2 S_{0} S_{1}^{3}\right) \alpha^{2} \\
& +\left(\frac{9}{4} S_{0}^{5}-\frac{233}{6} S_{0}^{4} S_{1}+\frac{262}{3} S_{0}^{3} S_{1}^{2}-\frac{152}{3} S_{0}^{2} S_{1}^{3}+\frac{20}{3} S_{0} S_{1}^{4}\right) \alpha^{3}+\mathcal{O}\left(\alpha^{4}\right)
\end{aligned}
$$

where we defined $\alpha \equiv g / m^{2}$. We also checked explicitly that the relation (A.1) holds. Substituting (A.25) into the DV relation (5.3), we obtain the superpotential (5.5).

The $S p(N)$ result is obtained similarly, with the result as in (A.1).

\section{A.3. Computation of matrix model free energy: $S p(N)$ theory with antisymmetric tensor}

Consider the $S p(\mathbf{N})$ matrix model which corresponds to $S p(N)$ gauge theory with an antisymmetric tensor. The superpotential is taken to be quartic (5.12). The matrix variable $\boldsymbol{\Phi}$ satisfies $\boldsymbol{\Phi}=\mathbf{A} J, \mathbf{A}^{T}=-\mathbf{A}$. The "action" $W_{\text {tree }}$ is given in (5.12). By a complexified $S p(\mathbf{N})$ gauge rotation, the matrix $\mathbf{\Phi}$ can be brought to the form 40]

$$
\Phi \cong \operatorname{diag}\left[\lambda_{1}, \ldots, \lambda_{\mathbf{N}}\right] \otimes \mathbf{1}_{2}, \quad \lambda_{i} \in \mathbf{C}
$$

By changing the integration variables from $\Phi$ to $\lambda_{i}$, we obtain

$$
Z \sim \int \prod_{i=1}^{\mathbf{N}} d \lambda_{i} \prod_{i<j}^{\mathbf{N}}\left(\lambda_{i}-\lambda_{j}\right)^{4} e^{-\frac{1}{\mathrm{~g}} \sum_{i=1}^{\mathbf{N}}\left(\frac{m}{2} \lambda_{i}^{2}+\frac{g}{3} \lambda_{i}^{3}\right)}
$$

where $\prod_{i<j}^{\mathbf{N}}\left(\lambda_{i}-\lambda_{j}\right)^{4}$ comes from the Jacobian for this change of variables. The polynomial $\frac{m}{2} \lambda^{2}+\frac{g}{3} \lambda^{3}$ has two critical points $z=0,-\frac{m}{g}$, around which we would like to do perturbative expansion. For this purpose, we separate $\lambda$ 's into two groups as

$$
\lambda_{i}= \begin{cases}\lambda_{i_{0}}^{(1)} & i_{1}=1, \ldots, \mathbf{N}_{1} \\ -m / g+\lambda_{i_{1}}^{(2)} & i_{2}=1, \ldots, \mathbf{N}_{2}\end{cases}
$$


with $\mathbf{N}_{1}+\mathbf{N}_{2}=\mathbf{N}$. This corresponds to the classical supersymmetric vacuum with breaking pattern $S p(\mathbf{N}) \rightarrow S p\left(\mathbf{N}_{1}\right) \times S p\left(\mathbf{N}_{2}\right)$.

The matrix integral can be performed just the same way as for the $S O / S p(N)$ theory with adjoint, as described in the last subsection. After substitution $S_{1,2}=2 \mathbf{g N}_{1,2}$, we obtain

$$
\begin{aligned}
\mathcal{F}_{S^{2}}= & \left(-\frac{S_{1}^{3}}{3}+\frac{S_{2}^{3}}{3}+\frac{5}{2} S_{1}^{2} S_{2}-\frac{5}{2} S_{1} S_{2}^{2}\right) \alpha \\
& +\left(-\frac{4}{3} S_{1}^{4}+\frac{91}{6} S_{1}^{3} S_{2}-\frac{59}{2} S_{1}^{2} S_{2}^{2}+\frac{91}{6} S_{1} S_{2}^{3}-\frac{4}{3} S_{2}^{4}\right) \alpha^{2} \\
& +\left(-\frac{28}{3} S_{1}^{5}+\frac{871}{6} S_{1}^{4} S_{2}-\frac{1318}{3} S_{1}^{3} S_{2}^{2}+\frac{1318}{3} S_{1}^{2} S_{2}^{3}-\frac{871}{6} S_{1} S_{2}^{4}+\frac{28}{3} S_{2}^{5}\right) \alpha^{3}+\mathcal{O}\left(\alpha^{4}\right),
\end{aligned}
$$

where $\alpha \equiv g^{2} / m^{3}$. We also checked explicitly that the relation (A.1) holds. Substituting (A.29) into (5.14), we obtain the glueball superpotential (5.16).

\section{Appendix B. Gauge theory calculation of superpotential}

In this appendix, we compute the exact superpotential of the $\mathcal{N}=1 S O(2 N) / S p(N)$ theory with adjoint in various vacua by considering factorization of the $\mathcal{N}=2$ curve. This factorization method was developed in [3] for $U(N)$, and generalized in [48] to the case with unoccupied critical points (in other words, the $n<K$ case below). Inclusion of fundamentals was considered in [49]. The generalization to $S O / S p$ gauge group, which discuss below, was given in [29, 38, 50, 51, 52, [12].

First consider $\mathcal{N}=2 S O(2 N)$ theory broken to $\mathcal{N}=1$ by the following polynomial tree level superpotential for the adjoint chiral superfield $\Phi$ :

$$
\begin{aligned}
& W_{\text {tree }}=\frac{1}{2} \operatorname{Tr}[W(\Phi)], \\
& W(x)=\sum_{j=1}^{K+1} \frac{g_{2 j}}{2 j} x^{2 j}, \quad W^{\prime}(x)=g_{2 K+2} x \prod_{i=1}^{K}\left(x^{2}-a_{i}^{2}\right) .
\end{aligned}
$$

The classical supersymmetric vacua are obtained by putting $2 N_{0}$ eigenvalues of $\Phi$ at $x=0$ and $N_{i}$ pairs of eigenvalues at $x= \pm a_{i}$, where $i=1, \cdots, K$ and $N_{0}+\sum_{i=1}^{K} N_{i}=N$. In this vacuum the gauge group breaks as $S O(2 N) \rightarrow S O\left(2 N_{0}\right) \times \prod_{i=1}^{K} U\left(N_{i}\right)$. We allow some of $N_{i}$ to vanish, i.e. we allow "unoccupied" critical points. Let the number of nonzero $N_{i \geq 1}$ be $n$. Then, the $\mathcal{N}=2$ curve governing this $S O(2 N)$ theory factorizes as 29, 38,50]:

$$
y^{2}=P_{2 N}^{2}(x)-4 x^{4} \Lambda^{4 N-4}= \begin{cases}{\left[x H_{2 N-2 n-2}(x)\right]^{2} F_{2(2 n+1)}(x)} & N_{0}>0, \\ H_{2 N-2 n}(x)^{2} F_{4 n}(x) & N_{0}=0 .\end{cases}
$$


Here $P, H$ and $F$ are polynomials in $x$ of the subscripted degree, which are invariant under $x \rightarrow-x$, i.e. they are actually polynomials in $x^{2}$. This factorization is required to have the appropriate number of independent, massless, monopoles and dyons, which must condense to eliminate some of the low-energy photons. The polynomial $F$ is related to the tree level superpotential as

$$
\begin{cases}F_{2(2 n+1)}(x)=\frac{1}{g_{2 K+2}^{2}} W_{2 K+1}^{\prime}(x)^{2}+f_{2 K}(x) & n=K \\ F_{2(2 n+1)}(x) Q_{2 K-2 n}(x)^{2}=\frac{1}{g_{2 K+2}^{2}} W_{2 K+1}^{\prime}(x)^{2}+f_{2 K}(x) & n<K\end{cases}
$$

with some polynomial $Q_{2 K-2 n}(x), f_{2 K}(x)$ of the subscripted degrees, and $W_{2 K+1}^{\prime}(x)$ is as in (2.6). Equation (B.3) is for $N_{0}>0$, and for $N_{0}=0$ one must use the second equation with $Q_{2 K-2 n}$ replaced by $Q_{2 K-2 n+2}$.

For $N_{0}>0$ we can write the solution of (B.2) in terms of that of the corresponding $U(2 N-2)$ breaking pattern, via:

$$
P_{2 N}^{S O(2 N)}(x)=x^{2} P_{2 N-2}^{U(2 N-2)}(x) .
$$

The low energy superpotential is given by

$$
W_{\text {low }}=\frac{1}{2} \sum_{j=1}^{K+1} g_{2 j}\left\langle u_{2 j}\right\rangle
$$

where the $\left\langle u_{2 j}\right\rangle$ are constrained to satisfy (B.2). Implementing this leads to the result that |51]:

$$
\left\langle\operatorname{Tr} \frac{1}{x-\Phi}\right\rangle=\frac{d}{d x} \ln \left[P_{2 N}(x)+\sqrt{P_{2 N}(x)^{2}-4 x^{4} \Lambda^{4 N-4}}\right] .
$$

Plugging back into $(\mathbb{B} .5)$ gives $W_{\text {low }}$. Note that the superpotential takes this simple form (B.5) only after one integrates out the monopoles and dyons, whose equation of motion led to the factorization constraint (B.2) [53,54,21].

The $S p(N)$ theory can be solved similarly. The $\mathcal{N}=2$ curve factorizes in the vacuum with breaking pattern $S p(N) \rightarrow S p\left(N_{0}\right) \times \prod_{i=1}^{K} U\left(N_{i}\right)$ as 29, 38, 50.

$$
\begin{aligned}
y^{2} & =B_{2 N+2}(x)^{2}-4 \Lambda^{4 N+4}=x^{2} H_{2 N-2 n}(x)^{2} F_{2(2 n+1)}(x), \\
B_{2 N+2}(x) & \equiv x^{2} P_{2 N}(x)+2 \Lambda^{2 N+2}
\end{aligned}
$$

The polynomial $F_{2(2 n+1)}(x)$ is related to $W^{\prime}(x)$ by $($ B.3. $)$. The mapping of the $S p(N)$ theory to a $U(2 N+2)$ theory, as in (3.16), can be written as a solution of (B.7) in terms of solutions of the corresponding $U(2 N+2)$ factorization problem:

$$
B_{2 n+2}(x) \equiv x^{2} P_{2 N}^{S p(N)}(x)+2 \Lambda^{2 N+2}=P_{2 N+2}^{U(2 N+2)}(x) .
$$


The $\Lambda^{2 N+2}$ shift in (B.8) is an $S p(N)$ residual instanton effect, associated with the index of the embedding of the $U\left(N_{i}\right)$ factors in $S p(N)$ [55,43,

Again, the superpotential is given as in (B.5), subject to the constraint that $\left\langle u_{j}\right\rangle$ satisfy (B.7). Implementing this, the $\left\langle u_{j}\right\rangle$ can be obtained from $B_{2 N+2}(x)$ by [51]

$$
\left\langle\operatorname{Tr} \frac{1}{x-\Phi}\right\rangle=\frac{d}{d x} \ln \left[B_{2 N+2}(x)+\sqrt{B_{2 N+2}(x)^{2}-4 \Lambda^{4 N+4}}\right] .
$$

We now consider the exact $W_{\text {eff }}$ for a few $S O / S p(N)$ cases, to illustrate and clarify the general features 6 . We take quartic tree level superpotential

$$
W_{\text {tree }}=\frac{1}{2} \operatorname{Tr} W(\Phi), \quad W(x)=\frac{m}{2} x^{2}+\frac{g}{4} x^{4},
$$

which corresponds to $K=1$.

\section{B.1. $S O(2 N)$ unbroken}

By the map (3.14), this maps to $U(2 N-2)$ unbroken, for which $P^{U(2 N-2)}(x)=$ $2 \Lambda^{2 N-2} T_{2 N-2}(x / 2 \Lambda)$, with $T_{N}\left(x=\frac{1}{2}\left(t+t^{-1}\right)\right)=\frac{1}{2}\left(t^{N}+t^{-N}\right)$ a Chebyshev polynomial [56]. Thus, using (B.4), $P^{S O(2 N)}(x)=2 \Lambda^{2 N-2} x^{2} T_{2 N-2}(x / 2 \Lambda)$, as found in [21]. This then leads to 21]

$$
\left\langle u_{2 p}\right\rangle \equiv \frac{1}{2 p}\left\langle\operatorname{Tr} \Phi^{2 p}\right\rangle=\frac{2 N-2}{2 p}\left(\begin{array}{c}
2 p \\
p
\end{array}\right) \Lambda^{2 p} .
$$

In particular,

$$
\left\langle u_{2}\right\rangle=(2 N-2) \Lambda^{2}, \quad\left\langle u_{4}\right\rangle=3(N-1) \Lambda^{4},
$$

and the low-energy superpotential is $W_{\text {low }}=\frac{1}{2}\left(m\left\langle u_{2}\right\rangle+g\left\langle u_{4}\right\rangle\right)$ :

$$
W_{\text {low }}=(N-1)\left(m \Lambda^{2}+\frac{3}{2} g \Lambda^{4}\right) .
$$

\section{B.2. $S O(2 N) \rightarrow S O(2) \times U(N-1)$}

By the map (3.14), this maps to $U(2 N-2) \rightarrow U(0) \times U(N-1) \times U(N-1)$. Using (3.14), the multiplication map of [3] for the $U(2 N-2)$ theory leads to a similar multiplication map for the $S O(2 N)$ theory, which was discussed in [51]. Using this, we can construct the solution to the factorization problem for general $N$ in terms of that of say $N=2$, i.e. $S O(4) \rightarrow S O(2) \times U(1)$. In this case, equation $(\mathbb{B} .2)$ is

$$
y^{2}=P_{4}^{2}-4 x^{4} \Lambda^{4}=x^{2} F_{6} .
$$

\footnotetext{
6 More $S O / S p$ examples can be found in [51.
} 
The solution to this factorization problem is

$$
P_{4}=x^{2}\left(x^{2}-a^{2}\right), \quad F_{6}=x^{2}\left[\left(x^{2}-a^{2}\right)^{2}-4 \Lambda^{2}\right],
$$

from which we can see the breaking pattern $S O(4) \rightarrow S O(2) \times U(1)$. Using (B.6) gives

$$
u_{2}=a^{2}, \quad u_{4}=\frac{a^{4}}{2}+\Lambda^{4} .
$$

Further, the condition (B.3)

$$
F_{6}=\frac{1}{g^{2}} W_{3}^{\prime 2}+f_{2}
$$

leads to

$$
a^{2}=-\frac{m}{g}, \quad f_{2}=-4 \Lambda^{2} x^{2} .
$$

The solution for general $S O(2 N) \rightarrow S O(2) \times U(N-1)$, the multiplication map gives the solution to the factorization problem as $P_{2 N}(x)=2 x^{2} \Lambda^{2 N-2} T_{N-1}\left(\left(x^{2}-a^{2}\right) / 2 \Lambda^{2}\right)$, with $T_{N-1}$ the Chebyshev polynomial defined above. The effect is to rescale $u_{2}, u_{4}$, and hence $W_{\text {low }}$ by an overall factor of $N-1$ :

$$
W_{\text {low }}=(N-1)\left(-\frac{m^{2}}{4 g}+\frac{1}{2} g \Lambda^{4}\right) .
$$

This agrees with the result (5.10).

\section{B.3. $S O(4) \rightarrow U(2)$}

More generally, we could consider the breaking pattern $S O(2 N) \rightarrow U(N)$. The map of (3.14) is less useful here, when $N_{0}=0$, since it suggests mapping to $U(2 N-2) \rightarrow$ $U(-2) \times U(N) \times U(N)$ and the $U(-2)$ needs to be interpreted. In general, this breaking pattern leads to a complicated $W_{\text {low }}(\Lambda)$. We will here illustrate the case $S O(4) \rightarrow U(2)$, corresponding to $N=2, N_{0}=0, n=1, K=1$. Equation (B.2) is

$$
y^{2}=P_{4}^{2}-4 x^{4} \Lambda^{4}=H_{2}^{2} F_{4} .
$$

The solution to this factorization problem is

$$
P_{4}=\left(x^{2}-a^{2}\right)^{2}+2 \Lambda^{2} x^{2}, \quad H_{2}=x^{2}-a^{2}, \quad F_{4}=\left(x^{2}-a^{2}\right)^{2}+4 \Lambda^{2} x^{2} .
$$

In the classical $\Lambda \rightarrow 0$ limit, this shows $P_{4} \rightarrow\left(x^{2}-a^{2}\right)^{2}$, implying the breaking pattern $S O(4) \rightarrow U(2)$. (B.6) gives

$$
u_{2}=2\left(a^{2}-\Lambda^{2}\right), \quad u_{4}=\left(a^{4}-2 \Lambda^{2}\right)^{2}-\Lambda^{4} .
$$


Further, the condition (B.3)

$$
F_{4} x^{2}=\frac{1}{g^{2}} W_{3}^{\prime 2}+f_{2}
$$

leads to

$$
a^{2}=-\frac{m}{g}+2 \Lambda^{2}, \quad f_{2}=4 \Lambda^{2} x^{2}\left(-\frac{m}{g}+\Lambda^{2}\right) .
$$

Therefore the exact superpotential is

$$
W_{\text {low }}=-\frac{m^{2}}{2 g}+m \Lambda^{2}+\frac{1}{2} g \Lambda^{4} .
$$

B.4. $S p(2) \rightarrow U(2), S p(1) \times U(1)$

This corresponds to $N=2, n=1, K=1$. Equations (B.7) and (B.3) are

$$
y^{2}=B_{6}^{2}-4 \Lambda^{12}=x^{2} H_{2}^{2} F_{6}, \quad F_{6}=\frac{1}{g^{2}} W_{3}^{\prime 2}+f_{2} .
$$

This factorization problem is solved by [51]:

$$
P_{4}=\left(x^{2}-a^{2}\right)^{2}+\frac{4 \Lambda^{6}}{a^{4}}\left(x^{2}-2 a^{2}\right), \quad \frac{m}{g}=-a^{2}+\frac{4 \Lambda^{6}}{a^{4}}
$$

From (B.9), we obtain

$$
u_{2}=2 a^{2}-\frac{4 \Lambda^{6}}{a^{4}}, \quad u_{4}=a^{4}+\frac{8 \Lambda^{12}}{a^{8}} .
$$

This solution continuously connects two classically different vacua with breaking pattern $S p(2) \rightarrow U(2)$ and $S p(2) \rightarrow S p(1) \times U(1)$. Correspondingly there are two ways to take the classical limit: i) $\Lambda \rightarrow 0$ with $a$ fixed, and ii) $\Lambda, a \rightarrow 0$ with $w=2 \Lambda^{3} / a^{2}$ fixed. In these limits, $P_{4}(x)$ goes to i) $\left(x^{2}-a^{2}\right)^{2}$ and ii) $x^{2}\left(x^{2}+w^{2}\right)$, showing the aforementioned breaking pattern.

In the $S p(2) \rightarrow U(2)$ case, we solve the second equation of (B.27) with the condition $a^{2} \rightarrow-m / g$ as $\Lambda \rightarrow 0$. The solution is

$$
a^{2}=-\frac{m}{g}+\frac{4 g^{2} \Lambda^{6}}{m^{2}}+\frac{32 g^{5} \Lambda^{12}}{m^{5}}+\frac{448 g^{8} \Lambda^{18}}{m^{8}}+\cdots .
$$

From (B.28) and (B.5), one obtains the exact superpotential:

$$
W_{\text {low }}=-\frac{m^{2}}{2 g}-\frac{2 g^{2} \Lambda^{6}}{m}-\frac{4 g^{5} \Lambda^{12}}{m^{4}}-\frac{32 g^{8} \Lambda^{18}}{m^{7}}+\cdots .
$$

In the $S p(2) \rightarrow S p(1) \times U(1)$ case, we solve the second equation of (B.27) with the condition $w^{2} \rightarrow m / g$ as $\Lambda \rightarrow 0$. It is

$$
w=\frac{m^{1 / 2}}{g^{1 / 2}}+\frac{g \Lambda^{3}}{m}-\frac{3 g^{5 / 2} \Lambda^{6}}{2 m^{5 / 2}}+\frac{4 g^{4} \Lambda^{9}}{m^{4}}+\cdots .
$$

From (B.28) and (B.5), one obtains the exact superpotential:

$$
W_{\text {low }}=-\frac{m^{2}}{4 g}+2 m^{1 / 2} g^{1 / 2} \Lambda^{3}+\frac{g^{2} \Lambda^{6}}{m}-\frac{g^{7 / 2} \Lambda^{9}}{m^{5 / 2}}+\cdots .
$$




\section{References}

[1] R. Gopakumar and C. Vafa, "On the gauge theory/geometry correspondence," Adv. Theor. Math. Phys. 3, 1415 (1999) arXiv:hep-th/9811131.

[2] C. Vafa, "Superstrings and topological strings at large N," J. Math. Phys. 42, 2798 (2001) arXiv:hep-th/0008142.

[3] F. Cachazo, K. A. Intriligator and C. Vafa, "A large N duality via a geometric transition," Nucl. Phys. B 603, 3 (2001) arXiv:hep-th/0103067.

[4] R. Dijkgraaf and C. Vafa, "Matrix models, topological strings, and supersymmetric gauge theories," Nucl. Phys. B 644, 3 (2002) arXiv:hep-th/0206255.

[5] R. Dijkgraaf and C. Vafa, "A perturbative window into non-perturbative physics," arXiv:hep-th/0208048.

[6] R. Dijkgraaf, M. T. Grisaru, C. S. Lam, C. Vafa and D. Zanon, "Perturbative computation of glueball superpotentials," arXiv:hep-th/0211017.

[7] F. Cachazo, M. R. Douglas, N. Seiberg and E. Witten, "Chiral rings and anomalies in supersymmetric gauge theory," JHEP 0212, 071 (2002) arXiv:hep-th/0211170].

[8] R. Dijkgraaf and C. Vafa, "N = 1 supersymmetry, deconstruction, and bosonic gauge theories," arXiv:hep-th/0302011.

[9] M. Aganagic, K. Intriligator, C. Vafa and N. P. Warner, "The glueball superpotential," arXiv:hep-th/0304271.

[10] P. Kraus and M. Shigemori, "On the matter of the Dijkgraaf-Vafa conjecture," JHEP 0304, 052 (2003) arXiv:hep-th/0303104.

[11] F. Cachazo, "Notes on supersymmetric $\mathrm{Sp}(\mathrm{N})$ theories with an antisymmetric tensor," arXiv:hep-th/0307063.

[12] C. h. Ahn, B. Feng and Y. Ookouchi, "Phases of $\mathrm{N}=1 \mathrm{USp}(2 \mathrm{~N}(\mathrm{c}))$ gauge theories with flavors," arXiv:hep-th/0307190.

[13] K. Landsteiner and C. I. Lazaroiu, "On $\operatorname{Sp}(0)$ factors and orientifolds," arXiv:hepth/0310111.

[14] L. F. Alday and M. Cirafici, "Effective superpotentials via Konishi anomaly," JHEP 0305, 041 (2003) arXiv:hep-th/0304119.

[15] P. Kraus, A. V. Ryzhov and M. Shigemori, "Loop equations, matrix models, and N = 1 supersymmetric gauge theories," JHEP 0305, 059 (2003) arXiv:hep-th/0304138.

[16] M. Matone, "The affine connection of supersymmetric $\mathrm{SO}(\mathrm{N}) / \mathrm{Sp}(\mathrm{N})$ theories," JHEP 0310, 068 (2003) arXiv:hep-th/0307285.

[17] N. Dorey, T. J. Hollowood, S. Prem Kumar and A. Sinkovics, "Exact superpotentials from matrix models," JHEP 0211, 039 (2002) arXiv:hep-th/0209089.

[18] V. Balasubramanian, J. de Boer, B. Feng, Y. H. He, M. x. Huang, V. Jejjala and A. Naqvi, "Multi-trace superpotentials vs. matrix models," Commun. Math. Phys. 242, 361 (2003) arXiv:hep-th/0212082. 
[19] H. Ita, H. Nieder and Y. Oz, "Perturbative computation of glueball superpotentials for SO(N) and USp(N)," JHEP 0301, 018 (2003) arXiv:hep-th/0211261.

[20] S. K. Ashok, R. Corrado, N. Halmagyi, K. D. Kennaway and C. Romelsberger, "Unoriented strings, loop equations, and $\mathrm{N}=1$ superpotentials from matrix models," Phys. Rev. D 67, 086004 (2003) arXiv:hep-th/0211291.

[21] R. A. Janik and N. A. Obers, "SO(N) superpotential, Seiberg-Witten curves and loop equations," Phys. Lett. B 553, 309 (2003) arXiv:hep-th/0212069.

[22] A. Klemm, K. Landsteiner, C. I. Lazaroiu and I. Runkel, "Constructing gauge theory geometries from matrix models," JHEP 0305, 066 (2003) arXiv:hep-th/0303032.

[23] S. G. Naculich, H. J. Schnitzer and N. Wyllard, "Cubic curves from matrix models and generalized Konishi anomalies," JHEP 0308, 021 (2003) arXiv:hep-th/0303268.

[24] S. G. Naculich, H. J. Schnitzer and N. Wyllard, "Matrix-model description of N = 2 gauge theories with non-hyperelliptic Seiberg-Witten curves," arXiv:hep-th/0305263.

[25] A. Strominger, "Massless black holes and conifolds in string theory," Nucl. Phys. B 451, 96 (1995) arXiv:hep-th/9504090.

[26] T. R. Taylor and C. Vafa, "RR flux on Calabi-Yau and partial supersymmetry breaking," Phys. Lett. B 474, 130 (2000) arXiv:hep-th/9912152.

[27] P. Mayr, "On supersymmetry breaking in string theory and its realization in brane worlds," Nucl. Phys. B 593, 99 (2001) arXiv:hep-th/0003198.

[28] S. Sinha and C. Vafa, "SO and Sp Chern-Simons at large N," arXiv:hep-th/0012136.

[29] J. D. Edelstein, K. Oh and R. Tatar, "Orientifold, geometric transition and large N duality for SO/Sp gauge theories," JHEP 0105, 009 (2001) arXiv:hep-th/0104037.

[30] K. Landsteiner, C. I. Lazaroiu and R. Tatar, "(Anti)symmetric matter and superpotentials from IIB orientifolds," arXiv:hep-th/0306236.

[31] C. Csaki, M. Schmaltz, W. Skiba and J. Terning, "Gauge theories with tensors from branes and orientifolds," Phys. Rev. D 57, 7546 (1998) arXiv:hep-th/9801207.

[32] K. Landsteiner, C. I. Lazaroiu and R. Tatar, "Chiral field theories from conifolds," arXiv:hep-th/0310052.

[33] E. Witten, "Baryons and branes in anti de Sitter space," JHEP 9807, 006 (1998) arXiv:hep-th/9805112.

[34] D. J. Gross and H. Ooguri, "Aspects of large N gauge theory dynamics as seen by string theory," Phys. Rev. D 58, 106002 (1998) arXiv:hep-th/9805129.

[35] S. S. Gubser and I. R. Klebanov, "Baryons and domain walls in an N = 1 superconformal gauge theory," Phys. Rev. D 58, 125025 (1998) arXiv:hep-th/9808075.

[36] R. Dijkgraaf and C. Vafa, "On geometry and matrix models," Nucl. Phys. B 644, 21 (2002) arXiv:hep-th/0207106.

[37] F. Ferrari, "On exact superpotentials in confining vacua," Nucl. Phys. B 648, 161 (2003) arXiv:hep-th/0210135. 
[38] H. Fuji and Y. Ookouchi, "Confining phase superpotentials for SO/Sp gauge theories via geometric transition," JHEP 0302, 028 (2003) arXiv:hep-th/0205301.

[39] R. Abbaspur, A. Imaanpur and S. Parvizi, " $\mathrm{N}=2 \mathrm{SO}(\mathrm{N}) \mathrm{SYM}$ theory from matrix model," JHEP 0307, 043 (2003) arXiv:hep-th/0302083.

[40] P. L. Cho and P. Kraus, "Symplectic SUSY gauge theories with antisymmetric matter," Phys. Rev. D 54, 7640 (1996) arXiv:hep-th/9607200.

[41] K. A. Intriligator, "New RG fixed points and duality in supersymmetric $\mathrm{SP}(\mathrm{N}(\mathrm{c}))$ and SO(N(c)) gauge theories," Nucl. Phys. B 448, 187 (1995) arXiv:hep-th/9505051.

[42] R. Dijkgraaf, S. Gukov, V. A. Kazakov and C. Vafa, "Perturbative analysis of gauged matrix models," arXiv:hep-th/0210238.

[43] C. Csaki and H. Murayama, "Instantons in partially broken gauge groups," Nucl. Phys. B 532, 498 (1998) arXiv:hep-th/9804061.

[44] F. Cachazo and C. Vafa, "N=1 and $\mathrm{N}=2$ geometry from fluxes," arXiv:hepth/0206017.

[45] C. Csaki, W. Skiba and M. Schmaltz, "Exact results and duality for $\operatorname{Sp}(2 \mathrm{~N})$ SUSY gauge theories with an antisymmetric tensor," Nucl. Phys. B 487, 128 (1997) arXiv:hep-th/9607210.

[46] S. Elitzur, A. Forge, A. Giveon, K. A. Intriligator and E. Rabinovici, "Massless Monopoles Via Confining Phase Superpotentials," Phys. Lett. B 379, 121 (1996) arXiv:hep-th/9603051.

[47] M. L. Mehta, "Random matrices," Academic Press (1991).

[48] F. Cachazo, N. Seiberg and E. Witten, "Phases of N $=1$ supersymmetric gauge theories and matrices," JHEP 0302, 042 (2003) arXiv:hep-th/0301006.

[49] V. Balasubramanian, B. Feng, M. x. Huang and A. Naqvi, "Phases of N = 1 supersymmetric gauge theories with flavors," arXiv:hep-th/0303065.

[50] B. Feng, "Geometric dual and matrix theory for SO/Sp gauge theories," Nucl. Phys. B 661, 113 (2003) arXiv:hep-th/0212010.

[51] C. h. Ahn and Y. Ookouchi, "Phases of $\mathrm{N}=1$ supersymmetric SO / Sp gauge theories via matrix model," JHEP 0303, 010 (2003) [arXiv:hep-th/0302150].

[52] C. h. Ahn, B. Feng and Y. Ookouchi, "Phases of $\mathrm{N}=1 \mathrm{SO}(\mathrm{N}(\mathrm{c}))$ gauge theories with flavors," arXiv:hep-th/0306068.

[53] N. Seiberg and E. Witten, "Electric - magnetic duality, monopole condensation, and confinement in N=2 supersymmetric Yang-Mills theory," Nucl. Phys. B 426, 19 (1994) [Erratum-ibid. B 430, 485 (1994)] arXiv:hep-th/9407087.

[54] N. Seiberg and E. Witten, "Monopoles, duality and chiral symmetry breaking in N=2 supersymmetric QCD," Nucl. Phys. B 431, 484 (1994) arXiv:hep-th/9408099].

[55] K. A. Intriligator and N. Seiberg, "Duality, monopoles, dyons, confinement and oblique confinement in supersymmetric $\mathrm{SO}(\mathrm{N}(\mathrm{c}))$ gauge theories," Nucl. Phys. B 444, 125 (1995) arXiv:hep-th/9503179.

[56] M. R. Douglas and S. H. Shenker, "Dynamics of SU(N) supersymmetric gauge theory," Nucl. Phys. B 447, 271 (1995) arXiv:hep-th/9503163. 